\title{
ZUR THEORIE
}

\section{DER TRIGONOMETRISCHEN REIHE}

VON

Prof. E. HOSSENFELDER.

WISSENSCHAFTLICHE BEILAGE ZUM XXVII. JAHRESBERICHT

DES KÖNIGL. GYMNASIUMS ZU STRASBURG (WESTPR.) ÜBER DAS SCHULJAHR 1899/1900.

DRUCK VON B. G. TEUBNER IN LEIPZIG.

1900. Progr. Nr. 40. 1900. 

Bei der Beschäftigung mit der Theorie der trigonometrischen Reihe und verwandten Gegenständen hatte ich bisweilen Gelegenheit zu bemerken, wie durch eine etwas modifizierte Darstellung in Ausdruck und Beweis gewisse überlieferte Theoreme an Anschaulichkeit und überzeugender Kraft gewinnen; ja es ergab sich bei einer zusammenhängenden Reihe von Sätzen über das Wünschenswerte hinaus die Notwendigkeit, zu Gunsten der Strenge und der damit zusammenhängenden Forderung, die Voraussetzungen möglichst einzuschränken, Änderungen eintreten zu lassen. Während ich in $\$ 1$ der vorliegenden Arbeit den ersten Satz aus Art. 8 der Riemann'schen Abhandlung über die Darstellbarkeit einer Funktion durch eine trigonometrische Reihe in einer andern Weise, als du BoisReymond, verallgemeinere, wodurch auch, abgesehen von der veränderten Darstellung, das Resultat ein etwas anderes, nämlich der in Rede stehende Limes in einen engeren Spielraum eingeschlossen wird, dienen die $\$ \S 2$ und 3 dazu, um die grundlegenden Sätze, die sich um den bekannten von Schwarz bewiesenen Hilfssatz vom zweiten Differentialquotienten gruppieren, nicht nur anschanlich darzustellen, sondern auch von gewissen wesentlichen Mängeln zu befreien, die sich, wie ich zeigen werde, bei du Bois-Reymond und Harnack vorfinden, wobei ich Gewicht daranf lege, dafs ich gerade den direkten vom ersten und dann auch vom zweiten der eben genannten Autoren eingeschlagenen Weg weiter verfolgt habe. - In $\$ 4$ gebe ich über das Verhältnis der Methode des arithmetischen Mittels des Herrn C. Neumann zur Fourier'schen Reihe eine ältere Aufzeichnung wieder (aus dem Jahre 1890), in welcher unter Vermeidung jeder weiteren Voraussetzung über die in Rede stehende darzustellende stetige Funktion gezeigt wird, wie die Fourier'sche Reihe ganz direkt aus der Methode des arithmetischen Mittels folgt. Einige hier sich ansehliefsende Bemerkungen haben zum Gegenstande die Beziehungen der Fourier'schen Reihe zum Poisson'schen Integral. -

\$1. Die Erweiterung des ersten Satzes aus Art. 8 der Riemann'schon Abhandlung in andrer Form.

Die zu Grunde liegende trigonometrische Reihe sei

$$
f(x)=\sum_{0}^{\infty}\left(a_{h} \cos h x+b_{h} \sin h x\right)=\sum_{0}^{\infty} A_{h} .
$$

Wir bilden mit Riemann die Reihe, welche aus dieser durch zweimalige Integration der einzelnen Glieder entsteht, und setzen

$$
F(x)=\frac{a_{0} x^{2}}{2}-\sum_{1}^{\infty} \frac{a_{h} \cos h x+b_{h} \sin h x}{h^{2}}=\frac{A_{0} x^{2}}{2}-\sum_{1}^{\infty} \frac{A_{h}}{h^{2}} .
$$

Dieselbe ist für alle Werte von $x$ gleichmälsig konvergent, falls die $A_{s}$ unter einer endlichen Zahl liegen, und stellt also eine stetige Funktion von $x$ dar. Wegen der gleichmäfsigen Konvergenz ist die Integration gliedweise gestattet. Nachdem festgestellt ist, dafs man sich bei dieser letzten Reihe auf völlig sicherem Boden befindet, handelt es sich darum, den Rückweg zu jener ersten zu finden, welche eigentlich zur Diskussion steht.

$\mathrm{Zu}$ dem Ende wird der zweite mittlere Differenzenquotient gebildet, also

$$
\frac{F(x+2 \varepsilon)-2 F(x)+F(x-2 \varepsilon)}{4 \varepsilon^{4}}=\frac{\Delta^{2} F}{4 \varepsilon^{2}} .
$$

$1^{\circ}$

Universitaits- und

Landesbibliothek Düsseldorf 
Man erhält:

$$
\frac{\Delta^{2} F}{4 \varepsilon^{2}}=A_{0}+\sum_{1}^{\infty} A_{\lambda}\left(\frac{\sin h \varepsilon}{h \varepsilon}\right)^{2}
$$

Der Riemann'sche Satz lautet:

Für jeden Wert $x$, für welchen die ursprïngliche Reihe konvergiert, konvergiert auch den $\operatorname{Lim}_{t=0} \frac{\Delta^{2} F}{4 \varepsilon^{2}}$, und swar nach demselben Werte.

Ich will nun die von P. du Bois-Reymond*) aufgeworfene und beantwortete Frage, was aus dem Riemann'schen Satze wird, wenn die Reihe $f(x)$ unbestimmt zwischen den Grenzen $U(x)$ und $O(x)$ angenommen wird, in etwas anderer Weise behandeln und beantworten.

Wenn es sich darum handelt,

$$
\operatorname{Lim}_{z=0} \frac{\Delta^{2} F}{4 z^{2}}=\operatorname{Lim}_{\varepsilon=0} \sum_{0}^{\infty} A_{h}\left(\frac{\sin h \varepsilon}{h \varepsilon}\right)^{2}
$$

zu bestimmen, so ist die Reihe bei von 0 verschiedenem positiven $\varepsilon$ ins Unendliche fortzusetzen und dann erst der Limes für $\varepsilon=0$ zu bilden. Andrerseits steht es frei, da man eine unendliche Reihe auf beliebige Weise in Partialsummen zerlegen kann, für jedes $\varepsilon$ eine andere Zerlegung vorzunehmen, d. h. also die Grenzen der Teilsummen bei dem Übergang zur Grenze $\varepsilon=0$ als von $\varepsilon$ abhängig auzunehmen.

Die Reihe $a_{0}+\sum_{0}^{\infty} a_{h} \cos h x+b_{h} \sin h x$ schwanke für den in Rede stehenden Wert von $x$ zwischen $O(x)$ und $U(x)$, sie sei also enthalten in der Form $\frac{O+U}{2}+j \frac{O-U}{2}$ (das $x$ lasse ich jetzt aus). Was lälst sich von dem $\operatorname{Lim}_{\varepsilon=0} \frac{\Delta^{2} F}{4 \varepsilon^{2}}$ aussagen? Während in dem Riemann'schen Falle an Stelle der Reste die Partialsummen ohne Änderung des Resultats hätten eingeführt werden können, ist in dem allgemeineren. Falle, wie er hier vorliegt, die Einführung der Summen vorzuziehen und zwar aus mehreren Gründen. Zunächst kann man hier sogleich die Gliederzahl der in Rede stehenden Reihe unendlich setzen, was bei du Bois-Reymond wegen der Unbestimmtheit der Reste der unendlichen Reihe nicht angeht (vgl. seine Abhandiung S. 18 oben); ferner schwanken die Summen schliefslich zwischen $O$ und $U$ (Differenz $O-U$ ), die Reste zwischen $O-U$ und $-(O-U$ ), also in doppelt so weiten Grenzen. Den Vorteil aber, welchen bei Multiplikation mit unbekannten Zahlen die Rechnung mit Null hat, bezw. mit Unbestimmtheitsgrenzen, welche gleichweit über und unter Null liegen, kann man sich bei Summen auch verschaffen, wenn man statt der obigen Reihen diejenigen untersucht, welche durch Subtraktion der Grösse $\frac{O+U}{2}$ vom Anfangsgliede $a_{0}$ oder $A_{0}$ entstehen. Nach Voraussetzung schwankt die Reihe

$$
\left(a_{0}-\frac{O+U}{2}\right)+\sum_{1}^{\infty} a_{A} \cos h x+b_{h} \sin h x=A_{0}^{\prime}+\sum_{1}^{\infty} A_{k}
$$

zwischen $\frac{O-U}{2}$ und $-\frac{O-U}{2}$; es wird gefragt, was hieraus für den

folgt, wo

$$
\operatorname{Lim}_{t=0} \frac{\Delta^{2} F^{\prime}}{4 \varepsilon^{2}}=\operatorname{Lim}_{\varepsilon=0}\left[A_{0}^{\prime}+\sum_{1}^{\infty} A_{h}\left(\frac{\sin h \varepsilon}{h \varepsilon}\right)^{2}\right]
$$

gesetzt ist?

$$
A_{0}^{\prime}=A_{0}-\frac{O+U}{2}=a_{0}-\frac{O+U}{2}
$$

Ich führe die Teilsummen der ursprünglichen Reihe ein und setze:

*) Abhandlungen der bayerischen Akademie d. W. II, CI, XII, Bd, I. Abt. 
$-5-$

$$
\begin{aligned}
& S_{0}=A_{0}^{\prime} \\
& S_{n}=A_{0}^{\prime}+\sum_{1}^{\prime \prime} A_{h},
\end{aligned}
$$

also

$$
A_{h}=S_{\Lambda}-S_{h-1} \text {, }
$$

so dass

$$
\begin{aligned}
A_{0}^{\prime}+\sum_{1}^{N} A_{h}\left(\frac{\sin h \varepsilon}{h \varepsilon}\right)^{2} & =S_{0}+\left(S_{1}-S_{0}\right)\left(\frac{\sin \varepsilon}{\varepsilon}\right)^{2}+\cdots+\left(S_{N}-S_{N-1}\right)\left(\frac{\sin N \varepsilon}{N \varepsilon}\right)^{2} \\
& =S_{0}\left(1-\left(\frac{\sin \varepsilon}{\varepsilon}\right)^{2}\right)+\cdots+S_{N-1}\left(\left(\frac{\sin (N-1) \varepsilon}{(N-1) \varepsilon}\right)^{2}-\left(\frac{\sin N \varepsilon}{N \varepsilon}\right)^{2}\right)+S_{N}\left(\frac{\sin N \varepsilon}{N \varepsilon}\right)^{2} .
\end{aligned}
$$

Wir lassen jetzt $N$ ins Unendliche wachsen: die Reihe links konvergiert jedenfalls für jedes $\varepsilon>0$, wie sowohl aus ihrer Entstehung aus der durchweg konvergenten Reihe für $F(x)$, als auch aus dem Faktor $h^{2}$ im Nenner jedes Gliedes und der Endlichkeit sämtlicher $A_{A}$ folgt. Demzufolge darf man auch rechts $N=\infty$ setzen und erhält, da bei der vorausgesetzten Endlichkeit sämtlicher $S_{N}$ $\lim _{N=\infty} S_{N}\left(\frac{\sin N \varepsilon}{N \varepsilon}\right)^{2}=0$ für jedes $\varepsilon>0$, folgende Gleichung:

$$
\frac{\Delta^{2} F^{\prime}}{4 \varepsilon^{2}}=A_{0}^{\prime}+\sum_{1}^{\infty} A_{h}\left(\frac{\sin h \varepsilon}{h \varepsilon}\right)^{2}=\sum_{0}^{\infty} S_{h}\left(\left(\frac{\sin h \varepsilon}{h \varepsilon}\right)^{2}-\left(\frac{\sin (h+1) \varepsilon}{(h+1) \varepsilon}\right)^{2}\right),
$$

wo $F^{\prime}$ aus $F$ entsteht, wenn man $A_{0}$ durch $A_{0}^{\prime}$ ersetzt. In dieser Reihe soll $\delta$ gegen 0 konvergieren. Ich mache die Riemann'sche Einteilung, welehe die Thatsache benutzt, dafs $\left(\frac{\sin \varepsilon}{\varepsilon}\right)^{\prime \prime}$ von 0 an bis $\pi$ monoton ist. Die Teilung richtet sich nach der Gröfse von $\varepsilon$ (s. oben); die erste Summe gehe von 0 bis $s$, wo $s \varepsilon<\pi \leq(s+1) \varepsilon$, die zweite von $s+1$ bis $\infty$. Die erste Summe erhalte eine Unterabteilung von 0 bis $m-1$ und von $m$ bis $s$, vobei $m$ sur Verfügung bleibt.

Hierdurch wird:

$$
A_{0}^{\prime}+\sum_{1}^{\infty} A_{h}\left(\frac{\sin h \varepsilon}{h \varepsilon}\right)^{2}=\sum_{0}^{m-1} S_{h}\left(\left(\frac{\sin h \varepsilon}{h \varepsilon}\right)^{2}-\left(\frac{\sin (h+1) \varepsilon}{(h+1) \varepsilon}\right)^{2}\right)+\sum_{m}^{\infty}+\sum_{s+1}^{\infty} .
$$

In den beiden ersten Summen rechter Hand ist die Funktion in der Klammer positiv, also der Mittelwertsatz anwendbar. Bezeichnen wir mit du Bois-Reymond durch $\left[S_{A}\right]_{0}^{m-1}$ einen Mittelwert der Grölsen $S_{0}, S_{1}, \ldots S_{m-1}$, entsprechend durch $\left[S_{h}\right]_{m}^{*}$ einen solchen der Grölsen $S_{m}, \ldots S_{*}$ u. s. w., so entsteht:

$$
\begin{aligned}
A_{0}^{\prime}+\sum_{1}^{\infty} A_{h}\left(\frac{\sin h \varepsilon}{h \varepsilon}\right)^{2} & =\left[S_{h}\right]_{0}^{m-1}\left(1-\left(\frac{\sin m \varepsilon}{m \varepsilon}\right)^{2}\right)+\left[S_{h}\right]_{m}^{2}\left(\left(\frac{\sin m \varepsilon}{m \varepsilon}\right)^{2}-\left(\frac{\sin (s+1) \varepsilon}{(s+1) \varepsilon}\right)^{2}\right) \\
& +\sum_{s+1}^{\infty} S_{h}\left(\left(\frac{\sin h \varepsilon}{h \varepsilon}\right)^{2}-\left(\frac{\sin (h+1) \varepsilon}{(h+1) \varepsilon}\right)^{2}\right) .
\end{aligned}
$$

Die letzte Summe zerlegen wir mit Riemann in folgender Weise:

$$
\left(\frac{\sin h \varepsilon}{h \varepsilon}\right)^{2}-\left(\frac{\sin (h+1) \varepsilon}{(h+1) \varepsilon}\right)^{2}=\left[\left(\frac{\sin h \varepsilon}{h \varepsilon}\right)^{2}-\left(\frac{\sin h \varepsilon}{(h+1) \varepsilon}\right)^{2}\right]+\left[\left(\frac{\sin h \varepsilon}{(h+1) \varepsilon}\right)^{2}-\left(\frac{\sin (h+1) \varepsilon}{(h+1) \varepsilon}\right)^{2}\right]
$$

und wenden die Formel an:

Hierdureh erhalten wir:

$$
\sin ^{2} x-\sin ^{2} y=\sin (x+y) \sin (x-y) .
$$

$$
\begin{aligned}
A_{0}^{\prime}+\sum_{1}^{\infty} A_{h}\left(\frac{\sin h \varepsilon}{h \varepsilon}\right)^{2} & =\left[S_{h}\right]_{0}^{m-1}\left(1-\left(\frac{\sin m \varepsilon}{m \varepsilon}\right)^{2}\right)+\left[S_{h}\right]_{m}\left(\left(\frac{\sin m \varepsilon}{m \varepsilon}\right)^{2}-\left(\frac{\sin (s+1) \varepsilon}{(s+1) \varepsilon}\right)^{2}\right) \\
& +\sum_{k+1}^{\infty} S_{h} \sin ^{2} h \varepsilon\left(\frac{1}{h^{2} \varepsilon^{2}}-\frac{1}{(h+1)^{2} \varepsilon^{2}}\right)-\sum_{s+1}^{\infty} S_{h} \frac{1}{(h+1)^{3} \varepsilon^{2}} \sin (2 h+1) \varepsilon \sin \varepsilon .
\end{aligned}
$$

Diese Gleichung gilt für jedes $\varepsilon>0$.

Universitäts- und

Landesbibliothek Düsseldorf 
Beim dritten und vierten Gliede kann nummehr auch der Mittelwertsatz angewandt werden, so dafs unsere Reihe wird

$$
\begin{aligned}
& =\left[S_{h}\right]_{0}^{m-1}\left(1-\left(\frac{\sin m \varepsilon}{m \varepsilon}\right)^{2}\right)+\left[S_{h}\right]_{m}^{s}\left(\left(\frac{\sin m \varepsilon}{m \varepsilon}\right)^{2}-\left(\frac{\sin (s+1) \varepsilon}{(s+1) \varepsilon}\right)^{2}\right)+\left[S_{h} \sin ^{2} h \varepsilon\right]_{s+1}^{\infty} \cdot \frac{1}{(s+1)^{2} \varepsilon^{2}} \\
& -\left[S_{h} \sin (2 h+1) \varepsilon \cdot \frac{\sin \varepsilon}{\varepsilon}\right]_{s+1}^{\infty} \cdot \frac{1}{\varepsilon}\left(\frac{1}{(s+2)^{2}}+\frac{1}{(s+3)^{2}}+\cdots\right),
\end{aligned}
$$

wo die Ausdrücke in den eckigen Klammern immer Mittelwerte sind. Es ist bekanntlich

$$
\frac{1}{(s+2)^{2}}+\frac{1}{(s+3)^{2}}+\cdots<\frac{1}{s+1}<\frac{1}{s}, \text { also }=\Theta_{s} \cdot \frac{1}{s},
$$

wo $\Theta_{x}<1$; somit erhalten wir:

$$
\begin{aligned}
\frac{\Delta^{2} F^{\prime}}{4 \varepsilon^{2}} & =\left[S_{h}\right]_{0}^{m-1}\left(1-\left(\frac{\sin m \varepsilon}{m \varepsilon}\right)^{2}\right)+\left[S_{h}\right]_{m}^{2}\left(\left(\frac{\sin m \varepsilon}{m \varepsilon}\right)^{2}-\left(\frac{\sin (s+1) \varepsilon}{(s+1) \varepsilon}\right)^{2}\right) \\
& +\left[S_{h} \sin ^{2} h \varepsilon\right]_{s+1}^{\infty} \cdot \frac{1}{(s+1)^{2} \varepsilon^{2}}-\left[S_{h} \sin (2 h+1) \varepsilon \cdot \frac{\sin \varepsilon}{\varepsilon}\right]_{s+1}^{\infty} \cdot \frac{1}{\varepsilon} \cdot \Theta_{s} \cdot \frac{1}{s} .
\end{aligned}
$$

Jetzt möge $\varepsilon$ zur 0 , also gleichzeitig $s$ gegen $\infty$ konvergieren. Links entsteht der zweite mittlere Differentialquotient der Funktion $F^{\prime}$. Die erste Klammer wird 0 für $\varepsilon=0$. In der zweiten Klammer wird das erste Glied 1, das zweite Glied wird, da sich der Festsetzung gemäfs $(s+1) \varepsilon$ bei kleiner werdendem $\varepsilon$ der Gröfse $\pi$ immer mehr nähert, 0 . Demgemäls wird

$$
\operatorname{Lim}_{\varepsilon=0} \frac{\Delta^{2} F^{\prime}}{4 \varepsilon^{2}}=\left[S_{h}\right]_{m}^{\infty}+\left[S_{h} \sin ^{2} h \varepsilon\right]_{\infty} \frac{1}{\pi^{2}}-\left[S_{h} \sin (2 h+1) \varepsilon \cdot \frac{\sin \varepsilon}{\varepsilon}\right]_{\infty} \Theta \cdot \frac{1}{\pi}
$$

wo $\Theta$ unbestimmt zwischen 0 und 1. Links kommt $m$ nicht vor; man darf daher rechts das zur Verfügung stehende $m$ beliebig und unendlich grofs werden lassen. Wenn man dann berücksichtigt, dass die $S_{h}$ für unendlich grofse $h$ der Voraussetzung nach zwischen $\frac{O-U}{2}$ und $-\frac{O-U}{2}$ schwanken, so erhält man:

$$
\operatorname{Lim}_{\varepsilon=0} \frac{\Delta^{2} F^{\prime}}{4 \varepsilon^{2}}=j \frac{O-U}{2}+j^{\prime} \frac{O-U}{2} \cdot \frac{1}{\pi^{2}}+j^{\prime \prime} \frac{O-U}{2} \cdot \frac{1}{\pi},
$$

wo $j, j^{\prime}, j^{\prime \prime}$ Faktoren objektiver Unbestimmtheit zwischen +1 und -1 bedeuten. Demzufolge ist, wie aus der Beziehung zwischen $F^{\prime}$ und $F$ folgt:

Wir haben also den Satz:

$$
\operatorname{Lim}_{\varepsilon=0} \frac{\Delta^{2} F}{4 \varepsilon^{2}}=\frac{O+U}{2}+j\left(1+\frac{1}{\pi}+\frac{1}{\pi^{2}}\right) \frac{O-U}{2} .
$$

Wenn die Reihe

$$
f(x)=\sum_{0}^{\infty} a_{h} \cos h x+b_{h} \sin h x
$$

swischen den endlichen Unbestimmtheitsgrensen $O(x)$ und $U(x)$ schwankt, so sind die Unbestimmtheitsgrenzen von

wo

$$
\operatorname{Lim}_{\varepsilon=0} \frac{F(x+\varepsilon)-2 F(x)+F(x-\varepsilon)}{\xi^{2}},
$$

enthalten zwischen

$$
F(x)=a_{0} \frac{x^{2}}{2}-\sum_{1}^{\infty} \frac{a_{h} \cos h x+b_{h} \sin h x}{h^{2}}
$$

und

$$
\begin{aligned}
& \frac{O(x)+U(x)}{2}+\left(1+\frac{1}{\pi}+\frac{1}{\pi^{2}}\right) \frac{O(x)-U(x)}{2} \\
& \frac{O(x)+U(x)}{2}-\left(1+\frac{1}{\pi}+\frac{1}{\pi^{2}}\right) \frac{O(x)-U(x)}{2}
\end{aligned}
$$


Über die Koeffizienten $A_{h}$ ist hierbei nur dasjenige vorausgesetzt, was aus der Oscillation der Reihe zwischen endlichen Grenzen folgt, nämlich dafs sie sämtlich numerisch unter einer endlichen Zahl liegen. Wenn die ursprüngliche Reihe, wie bei Riemann, konvergiert, so werden die Koeffizienten eo ipso als unendlich klein vorausgesetzt. Werden solche Voraussetzungen für ganze Intervalle gemacht, so übertragen sich nach dem Satze des Herrn G. Cantor die Angaben von den $A_{h}$, welche von $x$ abhängen, auf die $a_{h}$ und $b_{h}$, wie hier nur ganz im allgemeinen erinnert werden mag. Die in dem eben ausgesprochenen Satze angegebenen Grenzen sind natürlich durchaus nicht die du BoisReymond'schen Unbestimmtheitsgrenzen des in Rede stehenden Limes, doch sind diese letzteren sicherlich in dem angegebenen Spielraum enthalten. Die grölsere Unbestimmtheit rührt daher, dafs wir notgedrungen statt der Grenzen der rechten Seite die Grenzen der einzelnen Glieder der rechten Seite schätzten, wodurch sich die Unbestimmtheiten, welche sich unter Umständen in der Wirklichkeit wegheben, in der Rechnung summieren.

Es soll jetzt auch der Fall in Betracht gezogen werden, dafs die Summe der ursprünglichen Reihe unendlich wird, nehmen wir an $+\infty$. Führt man wiederum die Partialsummen ein, jetzt aber (ohne jene Grölse zu subtrahieren)

$$
\begin{aligned}
& S_{0}=A_{0}=a_{0} \\
& S_{n}=A_{0}+\sum_{1}^{n} A_{k}=\sum_{0}^{n} a_{h} \cos h x+b_{h} \sin h x,
\end{aligned}
$$

so darf man jetzt jenes Glied $S_{N}\left(\frac{\sin N_{\varepsilon}}{N_{\varepsilon}}\right)^{2}$ nicht für $N=\infty$ verschwinden lassen, sondern wird setzen dürfen, wobei die vorhergehenden Betrachtungen zum Teil in Kraft bleiben,

$$
\begin{aligned}
\frac{\Delta^{2} F}{4 \varepsilon^{2}} & =\left[S_{h}\right]_{0}^{m-1}\left(1-\left(\frac{\sin m \varepsilon}{m \varepsilon}\right)^{2}\right)+\left[S_{h}\right]_{m}^{s}\left(\left(\frac{\sin m \varepsilon}{m \varepsilon}\right)^{2}-\left(\frac{\sin (s+1) \varepsilon}{(s+1) \varepsilon}\right)^{2}\right)+S_{s}\left(\frac{\sin s \varepsilon}{s \varepsilon}\right)^{2} \\
& +\left[A_{h}\right]_{s}^{\infty} \sum_{s+1}^{\infty}\left(\frac{\sin h \varepsilon}{h \varepsilon}\right)^{2}=I+I I+I I I+I V .
\end{aligned}
$$

Läfst man wieder $\varepsilon$ gegen $0, s$ gegen $\infty$ konvergieren, so jedoch, dafs $s \varepsilon<\pi<(s+1) \varepsilon$ bleibt, so wird $I$ gegen 0 konvergieren, $I I$ gegen $\left[S_{h}\right]_{m}^{\infty}$, III gegen $\frac{1}{\pi^{2}} \lim _{s=\infty}\left(S_{s} \sin ^{2} s \varepsilon\right)$, IV gegen $\frac{1}{\pi^{2}} \lim _{s=\infty}\left\{s\left[A_{4}\right]_{s}^{\infty} \cdot \Theta_{s}\right\}$, wo $\Theta_{s}<1$.

In $I I$ darf man wieder die Grölse $m$, welche links nicht vorkommt, unendlich grofs werden lassen, so dafs $I I=+\infty$ wird nach unsrer jetzigen Voraussetzung; durch $I I I$, welches positiv ist, wird hieran nichts geändert, so dafs, wenn $I V$ endlich bleibt, sicherlich also, wenn $\lim _{s=\infty}\left(s a_{s}\right)$ und $\lim _{s=\infty}\left(s b_{s}\right)$ endlich bleiben, $\lim _{\varepsilon=0} \frac{\Delta^{2} F}{4 \varepsilon^{2}}=+\infty$ wird. Das Entsprechende gilt, wenn die Summe der Reihe $\sum_{0}^{\infty} A_{h}=-\infty$ wird.

Unter der Voraussetsung, da/s $\lim \left(n a_{n}\right)$ und $\lim \left(n b_{n}\right)$ für unendliche $n$ endlich bleiben, wird $\lim _{s=0} \frac{\Delta^{2} F}{4 \varepsilon^{2}}$ gleichseitig mit der Summe der ursprïnglichen trigonometrischen Reihe $+\infty$ bezw. $-\infty$.

Wenn $n a_{n}$ und $n b_{n}$ mit wachsendem $n$ unendlich klein werden, so bleibt übrig:

$$
\lim _{\varepsilon=0} \frac{\Delta^{2} F}{4 \varepsilon^{2}}=S_{\infty}+\frac{1}{\pi^{2}} \lim _{\substack{s=\infty \\ s t=\pi}}\left(S_{s} \sin ^{2} s \varepsilon\right)
$$

ohine jede Voraussetzung für $S_{\infty}$. Da das zweite Glied für endlich bleibende $S$ verschwindet, so folgt: $\lim _{t=0} \frac{\Delta^{y} F}{4 \varepsilon^{2}}$ schwankt, wenn $\lim \left(n a_{n}\right)=\lim \left(n b_{n}\right)=0$, mit der ursprünglichen Reihensumme swischen genau denselben Unbestimmtheitsgreneen $O$ und $U$, falls beide endlich sind. -

Ist $O=+\infty$ und $U$ endlich, so bleibt für dem Limes $+\infty$ als obere Unbestimmtheitsgrenze bestehen, während wegen des zweiten Gliedes die untere unter Umständen hinaufgerückt werden könnte. Das Entsprechende gilt, wenn $U=-\infty$ und $O$ endlich ist. - 


\section{§ 2. Gleichmäfsige Konvergenz. Funktionen vom Integral Null.}

Für das Intervall $a \leqq x \leq b$ sei eine aufserdem noch von dem Parameter $q$ abhängende Funktion $f(x, q)$ gegeben und es handle sich um den

$$
\lim _{q=+\infty} f(x, q) .
$$

Wenn für einen bestimmten Wert von $x$

$$
\left|f\left(x, q^{\prime}\right)-f\left(x, q^{\prime \prime}\right)\right|<\sigma,
$$

falls nur $q^{\prime}$ und $q^{\prime \prime}$ grölser als eine hinlänglich grofse Zahl $Q$ sind, so existiert für dieses $x$ ein Grenzwert. Findet entsprechendes für jedes $x$ statt, so erhält man eine Grenzfunktion

$$
\varphi(x)=\lim _{q=+\infty} f(x, q) .
$$

Wir können hierbei etwa an die Partialsummen einer unendlichen Reihe denken, deren Glieder Funktionen von $x$ sind: der Parameter $q$ ist dann die Gliederzahl $n$, welche ganzzahlig $=+\infty$ wird. Statt des Limes für $q=+\infty$ kann ein andrer eintreten, etwa der Limes für $\varepsilon=+0$ bei dem ersten Differenzenquotienten

$$
\frac{f(x+\varepsilon)-f(x)}{\varepsilon}
$$

welcher in den ersten vorderen Differentialquotienten, bezw. bei dem zweiten mittleren Differenzenquotienten

$$
\frac{f(x+\varepsilon)-2 f(x)+f(x-\varepsilon)}{\varepsilon^{2}},
$$

welcher in den zweiten mittleren Differentialquotienten übergeht, wenn ein solcher existiert. $q=+\infty$ repräsentiere also irgend einen Limes. - Die oben genannte Zahl $Q$ hängt im allgemeinen von $x$ ab. Wenn aber der besondere Fall eintritt, dafs für sämtliche $x$ gleichzeitig

$$
\left|f\left(x, q^{\prime}\right)-f\left(x, q^{\prime \prime}\right)\right|<\sigma
$$

für alle $q^{\prime}$ und $q^{\prime \prime}$, welche gröfser als ein bestimmtes hinlänglich grofses $Q$ sind, so sagt man bekanntlich, $f(x, q)$ konvergiere gleichmä/sig für das Intervall. - $f(x, q)$ sei im folgenden für jedes in Betracht kommende $q$ eine stetige Funktion von $x$,

Es kann die Frage aufgeworfen werden, aus welchen möglichst geringen Voraussetzungen bereits die gleichmäfsige Konvergenz der Funktion $f(x, q)$ für das ganze Intervall folgt. Hierauf antwortet folgender Satz:

Wenn $f(x, q)$ für jedes in Betracht kommende $q$ eine stetige Funlition von $x$ in dem Intervall $a \leq x \leq b$ ist, und wenn dieselbe für $q=+\infty$ gleichmälsig konvergiert für ein System von Punkten, velche überalldicht über das ganze Intervall verteilt sind, so konvergiert sie für alle $a \leq x \leq b$ gleichmä/sig.

Beweis: Das überall dichte Punktsystem, für welches n. V. die gleichmälsige Konvergenz stattfindet, sei das System $\xi ;$ die übrigen Punkte des Intervalls seien die Punkte $\eta$. Nach der Voraussetzung giebt es zu einem beliebig kleinen $\sigma>0$ eine Zahl $Q$, so dafs

$$
\left|f\left(\xi, q^{\prime}\right)-f\left(\xi, q^{\prime \prime}\right)\right|<\sigma \text {, }
$$

falls nur $q^{\prime}$ und $q^{\prime \prime}>Q$, und zovar für sämtliche है gleichzeitig.

Ist $\eta_{0}$ ein Punkt des Systems $\eta$, so ist wegen der vorausgesetzten Stetigkeit

$$
\left|f\left(x, q^{\prime}\right)-f\left(x, q^{\prime \prime}\right)-\left(f\left(\eta_{0}, q^{\prime}\right)-f\left(\eta_{0}, q^{\prime \prime}\right)\right)\right|<\sigma
$$

für alle $x$ innerhalb eines hinlänglich kleinen den Punkt $\eta_{0}$ umgebenden Intervalls $\delta$, (wo $\delta$ von $\eta_{0}, q^{\prime}, q^{\prime \prime}$ abhängen wird), gleichgiltig, ob diese $x$ nun zu den $\xi$ oder zu den $\eta$ gehören. Da es $\mathrm{n}$. V. in jedem beliebig kleinen Intervall Punkte $\xi$ giebt, so gilt (b) für gewisse $\xi$ mit (a) gleichzeitig, und es ergiebt sich durch Subtraktion

$$
\left|f\left(\eta_{0}, q^{\prime}\right)-f\left(\eta_{0}, q^{\prime \prime}\right)\right|<2 \sigma
$$

für $q^{\prime}, q^{\prime \prime}>Q$. Da hierin der Punkt $\eta_{0}$ irgend ein Punkt des Systems $\eta$ ist, so ist der ausgesprochene 
$-9-$

Satz bewiesen, denn man hat, um die Zahl $Q$ zu bestimmen, welche für sümtliche $x$ ausreicht, damit für $q^{\prime}, q^{\prime \prime}>Q$

$$
\left|f\left(x, q^{\prime}\right)-f\left(x, q^{\prime \prime}\right)\right|<\sigma
$$

wird, nur dasjenige $Q$ zu nehmen, welches für die Punkte $\xi$ die Annäherung

leistet.

$$
\left|f\left(\xi, q^{\prime}\right)-f\left(\xi, q^{\prime \prime}\right)\right|<\frac{\sigma}{2}
$$

Speziell gilt folgender Satz:

Wenn die Glieder einer Reihe $u_{0}(x), u_{1}(x), \ldots$ stetige Funktionen im Intervall $a \leq x \leq b$ sind, und wenn diese Reihe für ein überall dichtes Punltsystem gleichmä/sig lionvergiert, so lionvergiert sie für das ganze Intervall gleichmä/sig.

An Stelle von (a) und (b) treten beim Beweise dieses Satzes

$$
\left|u_{n+1}(\xi)+\cdots+u_{n+p}(\xi)\right|<\sigma, \quad p=0,1,2, \ldots,
$$

für alle $\xi$, wenn nur $n>\mu$, und

$$
\left|u_{n+1}(x)+\cdots+u_{n+p}(x)-\left(u_{n+1}\left(\eta_{0}\right)+\cdots+u_{n+p}\left(\eta_{0}\right)\right)\right|<\sigma
$$

für alle $x$ innerhalb eines hinlänglich kleinen Intervalls um $\eta_{0}$. Da unter diesen $x$ unter allen Umständen auch छे vorkommen, so entsteht durch Vereinigung für alle $n>\mu$

$$
\left|u_{n+1}\left(\eta_{0}\right)+\cdots+u_{n+p}\left(\eta_{0}\right)\right|<2 \sigma, \quad p=0,1,2, \ldots
$$

wobei $\eta_{0}$ ein beliebiger unter den Punkten $\eta$ ist*). -

Dieser Satz lehrt, welche Ausnatimen von der gleichförmigen Konvergenz auf der ganzen Strecke vorkommen können und welche nicht. Es kann thatsächlich nicht vorkommen, dals die Funktion $f(x, q)$ für sämtliche Punkte der Strecke mit Ausnahme der Punkte eines ausdehnungslosen (nach Harnack diskreten) Systems gleichmäfsig konvergiere; denn auf jeder beliebigen Teilstrecke befinden sich bekanntlich Punkte, die nicht zu dem ausgenommenen unausgedehnten System gehören.

Wirliche Ausnahmen von der gleichmä/sigen Konvergens im ganzen Intervalle liönnen nur in Strecken vorkommen: so kann es geschehen, dafs die Funktion nach Ausschlufs einer beliebig kleinen den Endpunkt enthaltenden Strecke gleichmäfsig konvergiere (Potenzreihe), oder die gleichmäfsige Konvergenz kann statthaben nach Aussehluls eines unausgedehnten Systems durch eine endliche Anzahl von Strecken mit beliebig kleiner Gesamtlänge $\varepsilon$. Wenn man in diesem Falle von einer im allgemeinen gleichmälsigen Konvergenz auf der ganzen Strecke sprechen will, so lälst sich dagegen nichts sagen, weil die ausgeschlossenen Strecken eine beliebig kleine Gesamtlänge $\varepsilon$ repräsentieren (bei kleiner werdendem $\varepsilon$ wird obiges $Q$ bei gegebenem $\sigma$ immer gröfser). Bei weitergehenden Ausnahmen von im allgemeinen gleichmälsiger Konvergenz zu sprechen dürfte sich nicht empfehlen. Hiervon wird noch weiter unten die Rede sein. -

Bekanntlich gilt folgender Satz

Wenn in dem Intervall $a \leqq x \leqq b \quad f(x, q)$ für alle in Betracht kommenden $q$ eine stetige Funktion von $x$ ist, welche für $q=+\infty$ gleichmä/sig sum Grenswert $\varphi(x)$ konvergiert, so ist $\varphi(x)$ in demselben eine stetige Funktion.

Daraus geht hervor, dafs, wenn die Grenzfunktion unstetig ist, von einer gleichmäfsigen Konvergenz nicht mehr die Rede sein kann, solange $f(x, q)$ als stetig voransgesetzt wird. In der Folge wird uns eine unstetige Funktion beschäftigen, welche Herr Pasch ${ }^{20 \%}$ ) nach Harnacks Vorgange im allgemeinen verschwindend nennt. Während ich sonst die in der zitierten Abhandlung gebrauchten Bezeichnungen für sehr treffend halte, möchte ich für die eben genannte Funktion die auch sonst angewandte Bezeichnung (Dini) "Funktion vom Integral Null für geeigneter halten. Diese Funktion ist nämlich so beschaffen, dafs das Integral derselben zwischen zwei beliebigen Punkten genommen verschwindet; sie erfüllt folgende Bedingung: Für jedes $\sigma>0$ müssen die Stellen mit abs $y>\sigma$ eine

2) Ein ganz spezieller Fall dieses Satzes findet sich bei Stolz, Arithmetik I, S. 273.

o*) Stolz, Arithm. I, S, 200.

*ats) Math. Ann, 30, S. 151.

2

Universitäts- und

Landesbibliothek Düsseldorf 
unausgedehnte Menge bilden. Sie kann auch definiert werden als eine Funktion, welche Integration zulälst und in überall dichten Punkten verschwindet. Hierbei ist, zunächst wenigstens, immer vorausgesetzt, dafs die Funktionswerte zwischen endlichen Grenzen sich befinden. - Ohne den Charakter einer solchen Funktion - als Funktion vom Integral Null - zu ändern, darf man bei ihr (nicht bei anderen integrierbaren Funktionen) beliebige Werte durch irgend welche absolut genommen kleinere ersetzen. Verbindet man diese Bemerkung mit der andern, dafs man die Funktion mit einer beliebig grofsen Konstante multiplizieren darf, so folgt daraus, dafs es frei steht, dieselbe mit einer durchweg endlichen, wenn auch an und für sich nicht integrierbaren Funktion zu multiplizieren. Zu bemerken ist, dafs die Sprïnge einer integrierbaren Funktion in den Punkten des betr. Intervalls der Definition nach eine Funktion vom Integral Null liefern. - Bei bestimmt gegebenem $\sigma$ kann man die Stellen mit abs $y>\sigma$ durch Intervalle mit beliebig kleiner Gesamtlänge ausschliefsen. Bei kleiner werdendem $\sigma$ kommen immer mehr auszuschliefsende Punkte neu hinzu. Trotzdem kann nach der Definition die Summe der ausschliefsenden Strecken, die ja bei jedem $\sigma$ beliebig klein gemacht werden kann, gleichzeitig immer mehr verkleinert werden, wobei aber die Anzahl dieser ausschliefsenden Strecken, jedesmal endlich, immer grölser wird und die Stellen, an denen sie auftreten, immer enger aneinander rücken. Dies wurde hier etwas umständlich ausgeführt, weil es bei dem Beweise der folgenden Sätze wesentlich ist. -

Es ist nicht nötig, dafs die einer Integration zu unterwerfende Funktion allenthalben einen bestimmten Wert habe. Da die Begriffe "Schwankung", "Sprung" ihre Bedeutung beibehalten, wenn die Funktion in den einzelnen Punkten zwischen endlichen Grenzen unbestimmt ist, so ergiebt sich, daf's eine so gegebene Funktion (etwa die Summe einer unendlichen Reihe) sehr wohl dieselbe Integrierbarkeitsbedingung erfüllen kann; natürlich mufs an den Stetigkeitsstellen, die überall dicht vorhanden sind, je ein bestimmter Wert vorliegen. Ist $\varphi(x)+j \psi(x)$ eine solche Funktion, wo $j$ der zwischen +1 und -1 liegende Du Bois-Reymond'sche Unbestimmtheitsfaktor ist, so ist das stets positive $\psi(x)$ eine Funktion vom Integral Null. Die Funktionen

$$
\varphi(x)+j \psi(x)=\frac{O(x)+U(x)}{2}+j \frac{O(x)-U(x)}{2}, \varphi(x)+\psi(x)=O(x), \quad \varphi(x)-\psi(x)=U(x)
$$

ergeben dasselbe Integral*). Umgekehrt wenn von den beiden Gröfsen $O(x)$ und $U(x)$ nur die eine integrierbar ist, oder wenn beide integrierbar sind und verschiedene Integrale liefern, dann ist die Funktion $\varphi(x)+j \psi(x)$ nicht integrierbar. Wenn aber bei einer stetigen Funktion die eine, etwa die vordere obere Derivierte (obere Unbestimmtheitsgrenze des vorderen Differenzenquotienten $\frac{f(x+\varepsilon)-f(x)}{\varepsilon}$ für $\varepsilon=+0$ ) integrierbar ist, so ist auch die vordere untere Derivierte, (sowie auch jede hintere), integrierbar und liefert dasselbe Integral; - es ist gleichgiltig, ob man die obere oder die untere Unbestimmtheitsgrenze oder die zwischen beiden unbestimmte Funktion der Integration unterwirft.

\section{§ 3. Verallgemeinerung des Schwarz'schen Satzes vom zweiten mittleren Differentialquotienten.} Entsprechendes vom ersten Differentialquotienten. dafs man in

Die seit dem Erscheinen der Riemann'schen Abhandlung angewandte Analyse besteht darin,

$F(x)$ dureh

$$
F(x)=\frac{a_{0} x^{2}}{2}-\sum_{1}^{\infty} \frac{a_{h} \cos h x+b_{h} \sin h x}{h^{2}}
$$

$$
f(x)=a_{0}+\sum_{1}^{\infty} a_{h} \cos h x+b_{h} \sin h x
$$

auszudrücken sucht und alsdann durch Integration vorstehender Gleichung für $F(x)$ die Koeffizienten $a$ und $b$ bestimmt $*$ * $)$. Zunächst sei $f(x)$ von $-\pi$ bis $+\pi$ stets konvergent und aufserdem eine

*) Du Bois-Reymond a, a. 0. Art. 11.

**) Ebenda Art. 14. 
stetige Funktion von $x$. Alsdann ist, gleichgiltig ob man den zweiten mittleren oder einen andern zweiten Differentialquotienten meint,

$$
\frac{d^{2}}{d x^{2}} \int_{-\pi}^{x} d \alpha \int_{-\pi}^{\alpha} d \beta f(\beta)=f(x)
$$

für alle Werte $-\pi<x<+\pi$. Desgleichen ist nach dem Riemann'schen Satze in diesem Falle

Setzt man jetzt

$$
\lim _{e=0} \frac{\Delta^{2} F}{\varepsilon^{2}}=f(x) \text {. }
$$

so folgt

$$
\Phi(x)=F(x)-\int_{-\pi}^{x} d \alpha \int_{-\pi}^{\alpha} d \beta f(\beta),
$$

$$
\lim _{\varepsilon=0} \frac{\Phi(x+\varepsilon)-2 \Phi(x)+\Phi(x-\varepsilon)}{\varepsilon^{2}}=0
$$

für $-\pi<x<+\pi$. Nach einem von Schwarz bewiesenen Hilfssatze*) folgt aus dieser Gleichung so dafs man setzen darf

$$
\Phi(x)=c_{0}+c_{1} x \text { für }-\pi \leqq x \leqq+\pi,
$$

$$
F(x)=\int_{-\pi}^{x} d \alpha \int_{-\pi}^{\alpha} d \beta f(\beta)+c_{0}+c_{1} x .
$$

Hieraus ergiebt sich (Art. 15 der du B.-R.sehen Abhdlg.) die Bestimmung der Koeffizienten in der Fourier'schen Form. -

Im allgemeinen Falle, dafs von $f(x)$ nur die Integrierbarkeit (zunächst im engeren Sinne bei durehgehender Endlichkeit) vorausgesetzt wird, was jedenfalls die Konvergenz der trigonometrischen Reihe in überall dichten Punkten verlangt, handelt es sich wieder um die Differenz

wo

$$
\Phi(x)=F(x)-F_{1}(x),
$$

Würde

$$
F_{1}(x)=\int_{-\pi}^{x} d \alpha \int_{-\pi}^{\alpha} d \beta f(\beta) .
$$

$$
\lim _{\varepsilon=0} \frac{\Delta^{2} \Phi}{\varepsilon^{2}}
$$

Null sein, so würde man ebenso weiter schliefsen dürfen. Dies lärst sich indessen nicht direkt beweisen. Was man direkt beweisen kann (ebenda Art. 17) ist, dafs dieser Limes bei der jetzigen Voraussetzung eine endliche Funktion vom Integral Null giebt. Wenn dies feststeht, so ist zu zeigen, dafs folgender Satz gilt:

Weifs man von einer für $a \leq x \leq b$ stetigen Funktion $\Phi(x)$, da/s ihr zweiter mittlerer Differentialquotient eine endliche Funktion $\nu(x)$ vom Integral Null ist, so ist für dasselbe Intervall

$$
\Phi(x)=\varepsilon_{0}+c_{1} x,
$$

d. h. der betr. Differentialquotient ist thatsächlich allenthalben $=0$. -

Ist dies bewiesen, - und um einen einwandfreien Beweis dieses Satzes unter Beibehaltung des du Bois-Reymond'schen Grundgedankens handelt es sich hier, - so ergeben sich wiederum die Koeffizienten in der bekannten Form der bestimmten Integrale. Wie ich schon in der Einleitung bemerkte, scheint mir der Beweis dieses Satzes in Art. 20 der öfter genannten Abhandlung nicht

*) Crelles J. 72, S. 141. Er lautet: Ist von einer stetigen Funktion der zweite mittlere Differentialquotient in einem Intervall Null, so ist daselbst die Funktion linear. 
zureichend, was ich weiter unten begründen werde. Im 24. Bande der Mathematisehen Annalen hat Harnack in seiner Abhandlung über die Prinzipien der Integralrechnung diesen Satz zunächst für den zweiten vorderen Differentialquotienten - und den entsprechenden für den ersten Differentialquotienten - neu formuliert und unter Benutzung des du Bois-Reymond'schen Grundgedankens wiederum bewiesen. Indessen die Formulierung des Satzes scheint mir wegen der darin enthaltenen überflüssigen Voraussetzung nicht glücklich und auch beim Beweise scheint ein näheres Eingehen auf die Art der Annäherung des betreffenden Differenzenquotienten an die Grenzfunktion, hier die Funktion vom Integral Null, geboten. Da der in Rede stehende Satz beim ersten Differentialquotienten sein vollständiges Analogon hat, so werde ich von diesem ausgehen und dabei, zu Gunsten der Anschaulichkeit, etwas weit ausholen. quotienten

Gegeben sei im Intervall $a \leqq x \leqq b$ eine endliche und stetige Funktion $f(x)$. Den Differenzen-

$$
\frac{f(x+\delta)-f(x)}{\delta}
$$

denke ich mir als Funktion zweier Variabeln, sie heifse $\psi(x, \delta)$, dargestellt: senkrecht zur $x$-Richtung die positive und negative $\delta$-Richtung. Durch die Werte der Funktion $f(x)$ von $a$ bis $b$ sind die Werte des vorwärts gebildeten Differenzenquotienten lediglich für das rechtwinklig gleichschenklige Argumentendreieck $a b c$, die Werte des rückwärts gebildeten für das kongruente Dreieck $a b c_{1}$ ge-

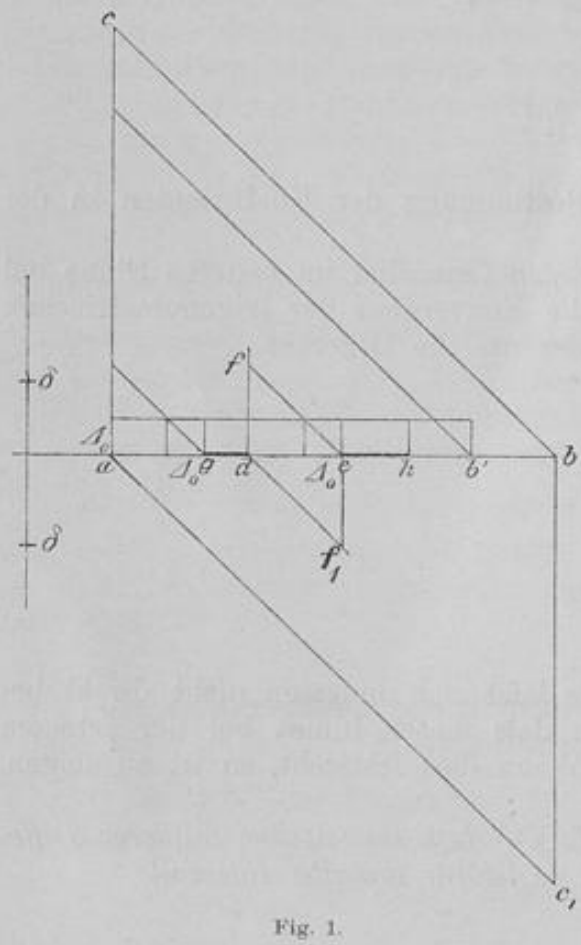
geben; man denke sich dieselben senkrecht aufgetragen. Da nach der Definition

$$
\psi(x, \delta)=\psi(x+\delta,-\delta),
$$

so finden sich in Punkten, wie $c$ und $c_{1}, f$ und $f_{1}$ u. s. w. die gleichen Funktionswerte. Für $\delta=+0$ entsteht bei einem bestimmten Werte von $x$ von oben her ein Grenzwert, der vordere Differentialquotient, oder ein zwischen zwei Unbestimmtheitsgrenzen, der vorderen oberen und unteren Derivierten, schwankender Limes; entsprechendes findet von unten her statt. Diese Grenzwerte bezw. Paare von Unbestimmtheitsgrenzen denke man sich auf der doppelt vorgestellten Linie aufgetragen; bei $b$ ist $\psi(b,+0)$, bei $a \psi(a,-0)$ nicht vorhanden. Folgendes*) ist zu beachten: Die untere bezw. obere Schranke der Differenzenquotienten ist zugleich untere bezw. obere Schranke jeder dex vier Derivierten. Hieraus geht hervor: der Wert in $c$, wie in dem ganzen Dreieck $a b c$ liegt zwischen der oberen und unteren Schranke der Werte längs $a b$, der Wert in $f$ zwischen den entsprechenden Schranken längs $d e$, die ganze Schwankung in einem Dreieck, wie def, kann nicht grölser sein, als längs de u. s. w.

$\psi(x, \delta)$ ist nach Ausschlufs eines beliebig schmalen Streifens zu beiden Seiten der $x$-Axe eine stetige Funktion beider Variabeln. Wenn allenthalben eine Derivierte im gewöhnlichen Sinne vorhanden ist, in $a$ eine vordere, in $b$ eine hintere, und wenn diese von $a$ bis $b$ einschliefslich der Grenzen stetig ist, so ist $\psi(x, \delta)$ eine im gansen Gebiete stetige Funktion beider Variabeln, was aus der jetzt vorausgesetzten Stetigkeit längs der $\delta$-Richtung einschliefslich $\delta=0$ und der Stetigkeit auf der ganzen Strecke $a b$ durchaus nicht allein folgt. Man kann nämlich wegen der Stetigkeit des Differentialquotienten für $a \leq x<b$ für ein gegebenes beliebig kleines $\sigma$ eine Länge $\Delta$ bestimmen, so dafs dieselbe längs $a b$ verschoben nur Schwankungen der Funktion $f^{\prime}(x)<\sigma$ enthält. Verschiebt man mit der Länge $\triangle$ die zugehörigen ein Parallelogramm bildenden rechtwinkligen Dreiecke (oben und unten), bezw. ein in dem Parallelo-

") Genaueres vgl. etwa Pasch, Math. Ann. Bd. 30, S, 138. 
$-13-$

gramm enthaltenes in Bezug auf die $x$-Axe symmetrisches Quadrat, so ist in jeder Lage die Schwankung im Quadrat, soweit es innerhalb des ursprünglichen Parallelogramms $a c b c_{1}$ liegt, $<\sigma$, woraus die Behauptung folgt*). -

Ich beweise jetzt unter Benutzung des du Bois-Reymond'schen Grundgedankens der Integralbildung den Satz:

Ist der vorwärts (rückwärts) gebildete erste Differentialquotient einer stetigen Funktion in einem Intervall eine endliche Funktion $v(x)$ vom Integral Null ${ }^{*}:$, so ist in demselben Intervall die gegebene stetige Funktion konstant, der Differentialquotient also durchiveg Null.

Beveis. Der Satz wird zurückgeführt auf den Dirichlet'schen Satz, nach welchem eine stetige Funktion, deren vorwärts gebildeter Differentialquotient Null ist, in demselben Intervall konstant sein mufs. Ich werde die Richtigkeit folgender Gleichung beweisen:

$$
\lim _{\delta=+0} \int_{a}^{\delta^{\prime}} \frac{f(\alpha+\delta)-f(\alpha)}{\delta} d \alpha=0, \quad a \leqq a^{\prime}<b^{\prime}<b .
$$

Das Integral wird also genommen längs der Parallelen zu $a b$ im Abstande $\delta$ (s. d. Fig. 1). N. V. lassen sich die Stellen auf $a b$, an denen abs $\nu(x)>\sigma$ ist, in eine bestimmte Anzahl Intervalle mit einer Gesammtlänge $<\delta$ einschliefsen $(g d$, eh repräsentieren von diesen Intervallen zwei auf einander folgende). Im zwischenliegenden Dreieck def und ebenso in den übrigen sind nach der obigen Auseinandersetzung die absoluten Werte durchweg, wie auf de, $\leqq$. Wenn man nun noch die kleinste der ausschliefsenden Strecken $\triangle_{0}$ links an jede einzelne derselben ansetzt und mit den andern ausschliefst, so ist nicht nur im Abstande $\Delta_{0}$, sondern auch in jedem kleineren $\delta$

$$
\int_{\varepsilon}^{\delta} \frac{f(\alpha+\delta)-f(\alpha)}{\delta} d c \mid<\sigma(b-a)+2 K_{\varepsilon}
$$

wo $\mathrm{K}$ eine Zahl ist, welche die absoluten Werte von $\nu(x)$ nicht überschreiten. Ist also eine beliebig kleine Zahl $\sigma_{0}$ willkürlich gegeben, so bestimme ich ein $\sigma$ aus $\sigma(b-a)<\frac{\sigma_{0}}{2}$, schliefse die Stellen mit abs $\nu(x)>\sigma$ in Intervalle mit einer Gesamtlänge $\varepsilon$, wo $2 \mathrm{~K} \varepsilon<\frac{\sigma_{0}}{2}$. Ist hierbei $\Delta_{0}$ das kleinste der ausschliefsenden Intervalle, so wird an jedes derselben links ein Intervall $\Delta_{0}$ angesetzt und mit den übrigen ausgeschlossen. Alsdann ist für alle positiven $\delta<\Delta_{0}$

$$
\int_{a}^{b^{\prime}} \frac{f(\alpha+\delta)-f(\alpha)}{\delta} d \alpha \mid<\sigma_{0}
$$

und zwar gleichmäfsig für alle $a \leqq a^{\prime}<b^{\prime}<b$. Wird $\sigma_{0}$ kleiner genommen, so findet man ein kleineres $\sigma$, ein kleineres $\varepsilon$, wobei aber die Anzahl der ausschliefsenden Intervalle wächst und dieselben an immer mehr Stellen, die vorher nicht ausgeschlossen waren, auftreten. Man sieht, dafs von einer angenommenen gleichmäfsigen Konvergenz der Funktion $\frac{f(x+\delta)-f(x)}{\delta}$ gegen die Grenzfunktion $\nu(x)$ nicht die Rede ist. Hiermit ist die obige Gleichung $(\dagger)$ bewiesen, welche sich auch in folgender Form schreiben lälst:

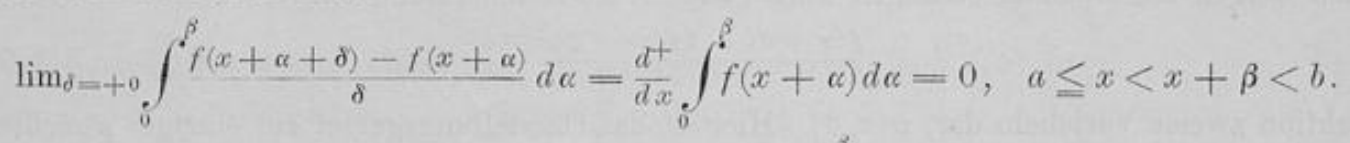

Nach dem Satze von Dirichlet ist nummehr das Integral $\int_{0}^{\beta} f(x+\alpha) d \alpha$, welches zugleich mit $f(x)$

*) Stolz, Diff.- u. Int.-Rchng. I, S. 55.

*it) oder ist, was dasselbe sagen will, eine der vier Derivierten eine Funktion vom Integral Null.

Universitaits- und

Landesbibliothek Düsseldorf 
eine stetige Funktion von $x$ ist, für $a \leq x<b$ von $x$ unabhängig, also eine Funktion von $\beta$ allein. $f(x)$ ist also gleich dem Differentialquotienten dieser Funktion von $\beta$ für $\beta=0$, also konstant und zwar wegen der Stetigkeit von $f(x)$ für $a \leq x \leq b$, w. z. b. w.

Alle diese Umstände sind nicht nötig, wenn es sich lediglich um einen möglichst kurzen Beweis des vorliegenden Satzes handelt*). Schliefst man wiederum die Stellen mit abs $v(x)>\sigma$ in Intervalle mit der Gesamtlänge $\varepsilon$, so wechseln sich ausschliefsende und andere Intervalle, in denen abs $\nu(x) \leq \sigma$, ab; sie seien der Reihe nach $\Delta_{1}, \Delta_{2}, \ldots$ Errichtet man auf $\Delta_{1}, \Delta_{2}, \ldots$ die rechtwinkligen Dreiecke, so ist nach den obigen Auseinandersetzungen der Wert des Differenzenquotienten in der Spitze abwechselnd $\leq \sigma$ und $<K$. Es gilt also

$$
\begin{aligned}
& f\left(a+\Delta_{1}\right)-f(a) \leqq \sigma \Delta_{1} \\
& f\left(a+\Delta_{1}+\Delta_{2}\right)-f\left(a+\Delta_{1}\right)<K \Delta_{2}
\end{aligned}
$$$$
f\left(a+\Delta_{1}\right)-f(a)<\mathrm{K} \Delta_{1}
$$$$
f\left(a+\Delta_{1}+\Delta_{2}\right)-f\left(a+\Delta_{1}\right) \leqq \sigma \Delta_{2}
$$

oder

je nachdem die Teilung mit einem gewöhnlichen Intervall oder einem ausschliefsenden beginnt. Jedenfalls ergiebt sich durch Addition

$$
f\left(b^{\prime}\right)-f(a)<\sigma(b-a)+\varepsilon K,
$$

d. h. $f\left(b^{\prime}\right)=f(a)$ für jedes $b^{\prime} \leq b$. -

Dieser Satz ergänzt den andern $*$ : Wenn von einer stetigen Funlition bekannt ist, da/s für alle $x$ zwischen $a$ und $\dot{b}$ eine der vier Derivierten Null ist mit Ausnahme einer abzählbaren Menge von Punkten, in denen ïber das Verhalten jener Derivierten nichts feststeht, so ist die Funktion eine Konstante.

$\mathrm{Zu}$ betonen ist aber, dafs bei unserm Satze die Endlichkeit der Derivierten eine wesentliche Voraussetzung ist, nicht so bei dem eben angeführten. Wenn z. B. der vordere Differentialquotient einer stetigen Funktion durchweg endlich und in allen Punkten mit Ausmahme eines ausdehnungslosen Systems Null ist, so ist die Funktion durchweg konstant. Läfst man die Voraussetzung der Endlichkeit fallen, so ist der Satz falsch, wie die von den Herren G. Cantor und Harnack ${ }^{\text {:kt:k }}$ ) aufgestellten nicht konstanten Funktionen zeigen, deren vorwärts gebildeter Differentialquotient allenthalben Null ist mit Ausnahme der Punkte eines ausdehnungslosen Systems, in welchen er unendlich ist. Hiergegen verstöfst auch der zweite eben angeführte Satz nicht, welcher die Endlichkeit nicht voraussetzt, weil in diesen Beispielen die Punkte des ausgenommenen (perfekten) Systems nicht abzählbar sind. Die beiden Sätze zeigen, welche Abweichungen von einer stetigen Funktion eine Derivierte nicht haben darf; wäre z. B.

$$
\frac{d^{+} f(x)}{d x}=\varphi(x)+v(x),
$$

wo $\varphi(x)$ eine endliche und stetige Funktion und $v(x)$ eine endliche Funktion vom Integral Null ist, so wäre

woraus $\nu(x)=0$ folgt.

$$
\frac{d^{+}}{d x}\left(f(x)-\int^{x} \varphi(x) d x\right)=\nu(x),
$$

Bei der Behandlung des sweiten mittleren Diffirensen- und Differentialquotienten kann ich mich nach den obigen Auseinandersetzungen kurz fassen. Auch hier stelle ich

$$
\frac{f(x+\delta)-2 f(x)+f(x-\delta)}{\delta^{2}}
$$

als Funktion zweier Variabeln dar, $\psi(x, \delta)$. Hier ist das Darstellungsgebiet ein einziges gleichschenklig rechtwinkliges Dreieck, dessen Hypotenuse jetzt aber das Stück der Abscissenaxe ist, für welches die stetige Funktion $f(x)$ definiert ist. Auf der Abscissenaxe finden sich die Werte des zweiten mittleren

*) Harnack, Math. Ann. 24, S. 235.

**) Dini, Grundlagen u. s. w. S. 274.

s*) Math. Ann. 24, S, 225. 
$-15-$

Differentialquotienten aufgetragen, wenn ein soleher existiert, andernfalls die beiden Unbestimmtheitsgrenzen. In $a$ und $b$ ist die Funktion nicht definiert. Auch hier ist der Wert der Funktion $\psi(x, \delta)$ in der Spitze $c$ des Dreiecks zwischen der oberen und unteren Schranke der Werte auf $a b$ gelegen, der Wert in $f$ zwischen der oberen und unteren Schranke auf de, kurz jeder Wert in einem solchen rechtwinkligen Dreieck nie aufserhalb der Werte auf der zugehörigen Hypotenuse:).

Beweis des Satses auf S. 11.

Dieser Satz ist durch Zurïckführung auf den Schwarz'schen Satz $\delta$ (S. 11 Fulsnote) ebenso zu erweisen, wie vorher der entsprechende vom ersten Differentialquotienten durch Zurückführung auf den Dirichletschen. Zunächst ist zu erweisen

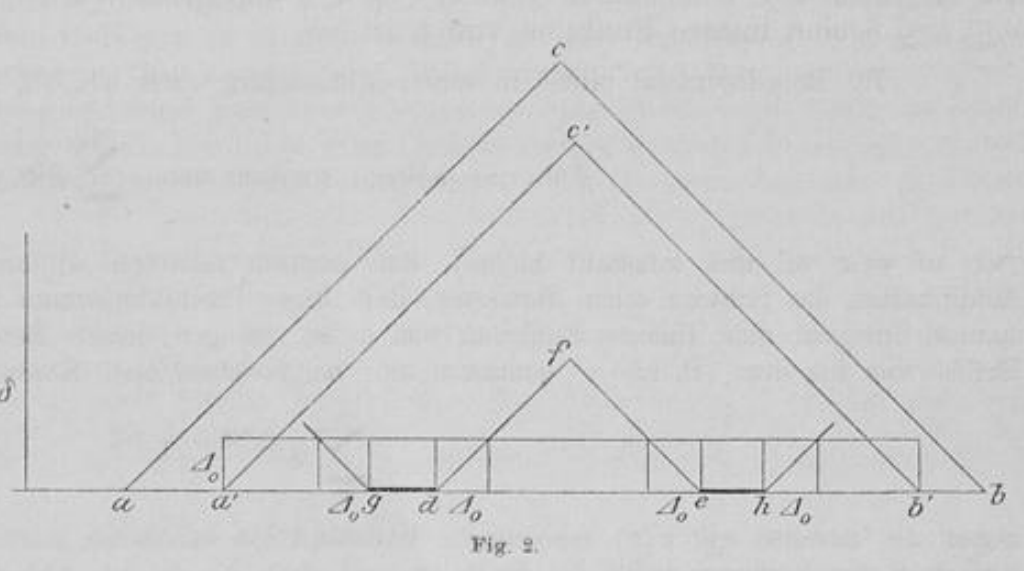

$$
\lim _{\delta=+0} \int_{\alpha^{\prime}}^{b^{\prime}} \frac{f(\alpha+\delta)-2 f(\alpha)+f(\alpha-\delta)}{\delta^{z}} d \alpha=0, \quad a<a^{\prime}<b^{\prime}<b .
$$

Wiederum schliefse man die Stellen mit abs $\nu(x)>\sigma$ in Intervalle mit der Gesamtlänge $\varepsilon$ ein, setze jetzt aber das kleinste der ausschliefsenden Intervalle $\Delta_{0}$ rechts und links an jedes einzelne derselben an und man erhält für alle $\delta<\Delta_{0}$

$$
\int \frac{f(\alpha+\delta)-2 f(\alpha)+f(\alpha-\delta)}{\delta^{2}} d \alpha \mid<\sigma(b-a)+3 K \varepsilon
$$

woraus wieder entsprechend wie oben (S. 13) die zu beweisende Gleichung folgt, welche auch in folgender Form geschrieben werden kann:

$$
\lim _{\delta=0} \int_{0}^{\beta} \frac{f(x+\alpha+\delta)-2 f(x+\alpha)+f(x+\alpha-\delta)}{\delta^{2}} d \alpha=\frac{d^{2}}{d x^{2}} \int_{0}^{\beta} f(x+\alpha) d \alpha=0, \quad a<x<x+\beta<b .
$$

Hierin bedeutet $\frac{d^{2}}{d x^{2}}$ den zweiten mittleren Differentialquotienten. Nach dem Satze von Schwarz ist demzufolge das Integral $\int_{0}^{\beta} f(x+\alpha) d \alpha$ eine lineare Funktion von $x$,

$$
\int_{0}^{\beta} f(x+\kappa) d \alpha=C_{0}+C_{1} x,
$$

wo $C_{0}$ und $C_{1}$ lediglich von $\beta$ abhängen. Wegen der Stetigkeit von $f(x)$ ist

$$
f(x)=\left[\frac{d}{d \beta}\left(C_{0}+C_{1} x\right)\right]_{\beta=0}
$$

und die rechte Seite mufs, wie die linke, für jedes $x$ völlig bestimmt sein. Daraus folgt, dafs die Differentialquotienten $\frac{d C_{0}}{d \beta}$ und $\frac{d C_{1}}{d \beta}$ für $\beta=0$ einzeln durehaus bestimmt sein müssen, weil sich Un-

*) Harnack, Math. Ann. 28, S. 269; Hölder, ebenda 24, S. 183.

Universitäts- und

Landesbibliothek Düsseldorf 
bestimmtheiten zweier Summanden nur bei gleicher Differenz der Unbestimmtheitsgrenzen möglicherweise aufheben können und eine solche Gleichheit bei veränderlichem $x$ hier nicht stattfinden kann. Da zu jedem $x<b$ unendlich viele $x+\beta<b$ angegeben werden können, so folgt, dafs $f(x)$ für alle $a \leqq x \leqq b$ eine lineare Funktion von $x$ ist.

Du Bois-Reymond bildet in seiner Abhandlung (Art. 18, 19, 20) nicht von

$$
\int_{0}^{\alpha} \Phi(x+\alpha) d \alpha, \text { sondern von } \sum_{0}^{n} \delta \Phi(x+p \delta),
$$

(wo $n \delta=a$ sei und konstant bleibe), den zweiten mittleren Differenzenquotienten und zeigt durch eine Modifikation des Schwarz'schen Bewreises, dafs diese Produktensumme für $\delta=0$, also dafs das soeben genannte Integral eine lineare Funktion von $x$ ist. Gegen diesen Beweis ist aber einzuwenden, dafs eine Gröfse wie die dort (S. 29) $\tau^{\prime}$ genannte, nur bei gleichmä/siger Konvergenz der Produktensumme

$$
\sum_{0}^{n} \delta \frac{\Delta=\Phi(x+p \delta)}{\varepsilon^{2}}
$$

gegen die daselbst mit $\tau(x)$ bezeichnete Grenzfunktion existieren kann, dafs von einer solehen bei der Unstetigkeit der letzteren nicht die Rede ist und dafs die in der Abhandlung gemachte Annahme, die unbestimmten Werte seien durch die numerisch grölstdenkbaren ersetzt, hieran nichts ändert. Harnack andrerseits giebt dem zu beweisenden entsprechenden Satze für den ersten Differentialquotienten eine andere Form*):

Ist für eine stetige Funltion $f(x)$, nach Ausschlu/s von Punlten durch Intervalle von endlicher Anzahl mit der Gesantlänge $\varepsilon$, an jeder Stelle $x$ ein und derselbe Wert von $\Delta x$ ausreichend, um die Ungleichung

$$
\frac{\Delta f(x)}{\Delta x}<\delta
$$

su erfüllen, wobei $\delta$ schliefslich unbegrenst Klein wird, ist forner $\lim \frac{\Delta f(x)}{\Delta x}$ durchaus endlich, und konvergicrt $\varepsilon$ mit $\triangle x$ nach Null, so ist $f(x)$ eine Constante.

Man vergleiche auch die Bemerkung hierzu S. 235. - Die Abänderung im Wortlaut des Satzes und im Beweise, die ich unter Beibehaltung des Grundgedankens vorgenommen habe, dürfte die Überflüssigkeit der ersten Voraussetzung ergeben und auch sonst den Beweis vervollstandigen. Entsprechendes gilt von dem Bexeise der Verallgemeinerung des Schwarz'schen Satzes; was den Wortlaut derselben anbelangt, so ist zu beachten, dafs auf S. 240, 241 der Harnack'sehen Abhandlung der zweite vordere Differentialquotient gemeint ist, und dafs auf S. 242 die Vereinfachung, welche beim zweiten mittleren gilt, im Einklang mit der vorliegenden Arbeit angegeben ist. - Herr Hölder hat den du Bois-Reymond'schen Satz auf einem neuen Wege bewiesen t*k $^{2}$ ). - -

\section{§ 4. Die C. Neumann'sche Methode des arithmetischen Mittels und die Fourier'sche Reihe. Das Poisson'sche Integral.}

Die Methode des arithmetischen Mittels, deren Urheber Herr C. Neumann ist, gewährt einen Ersatz für „das so schöne und dereinst so viel benutzte, jetzt aber wohl für immer dahingesunkene Dirichlet'sche Prinsip" ":*\$:) -

Versteht man unter einer Fundamentalfunktion eines ebenen von einer gegebenen Kurve $\sigma$ oder eines räumlichen von einer gegebenen Oberfläche $\sigma$ begrenzten Gebietes eine Funktion $U=U(x, y)$ bezw. $U=U(x, y, z)$, welche in Erstreckung des Gebietes eindeutig und stetig ist, deren erste und zweite Ableitungen nach $x, y$ bezw. $x, y, z$ innerhalb des Gebietes stetig sind und der Differentialgleichung genügen:

*) Math. Ann. 24, S. 233, 240, 248.

*) Math. Ann. 24, S. 181 .

C. Neumann, Abhandlungen der K. Sächsischen Gesellschaft d. W. Bd. XIII, 1887. Anfserdem ist be. sonders zu nennen das Werk desselben Verfassers über das Log. und Newton'sche Potential. Die letate mir bekannt gewordene Veroffentlichung über diese Methode ist enthalten in dem Bande XIV eben genannter Abhandlungen aus dem Jahre 1888 . 
$-17-$

$$
\frac{\partial^{2} U}{\partial x^{4}}+\frac{\partial^{2} U}{\partial y^{2}}=0, \text { bezw. } \frac{\partial^{2} U}{\partial x^{2}}+\frac{\partial^{2} U}{\partial y^{2}}+\frac{\partial^{2} U}{\partial z^{2}}=0,
$$

so löst die Methode zunächst für eine grofse Klasse von Kurven und Flächen die Aufgabe, diejenige Fundamentalfunktion eines gegebenen Gebietes zu finden, welche an der Begrenzung $\sigma$ gleich einer vorgeschriebenen als stetig angenommenen Funktion $f$ wird. Gleichzeitig wird bewiesen, dafs es nu. eine dem gegebenen Gebiet und der gegebenen Funktion $f$ entsprechende Fundamentalfunktion giebt. Von grundlegender Bedeutung für die Neumann'sche Theorie ist ein gewisses Potential, nämlich das Potential einer auf $\sigma$ ausgebreiteten Doppelbelegung vom Momente f. Dasselbe hat den Ausdruck

$$
W_{x}=\frac{1}{h \pi} \int \frac{\partial T}{\partial v} f d \sigma \text {, }
$$

wo $T$ in der Ebene $=\log \frac{1}{E}$, im Raume $=\frac{1}{E}$ ist, und $E$ den Abstand des Punktes $x$ von dem Elemente d $\sigma$ der begrenzenden Kurve oder Fläche bezeichnet. Die Ableitung $\frac{\partial T}{\partial v}$ erfolgt nach der inneren Normale. $h$ ist in der Ebene $=1$, im Raume $=2$. Dieses Potential kann in folgende Form gebracht werden:

$$
W_{x}=\frac{1}{h \pi} \int \frac{\cos \delta}{E^{h}} d \sigma
$$

wo $\delta$ den Winkel bezeichnet, unter welchem die Entfernung $E(d \sigma \rightarrow x)$ gegen die auf $d \sigma$ errichtete innere Normale geneigt ist, oder auch in folgende:

$$
W_{x}=\frac{1}{h \pi} \int f\left(d \sigma_{x}\right)
$$

wenn die scheinbare Grö/se des Elements $d \sigma$ für einen in $x$ befindlichen Beobachter mit $+\left(d \sigma_{x}\right)$ oder - $\left(d \sigma_{x}\right)$ bezeichnet wird, je nachdem der Beobachter die innere oder äufsere Seite des Elements vor Augen hat. Unterscheidet man zwischen $W_{i}$ und $W_{s}$, je nachdem Punkt $x$ im Innern von $\sigma$ oder auf $\sigma$ liegt, nennt ferner $W_{i s}$ den Konvergenswert, weleher auftritt, wenn in $W_{i}$ der Punkt $i$ in einen Punkt $s$ hineinrückt, so wird bewiesen, dals

wo

$$
\begin{gathered}
W_{i s}=f_{s}+f_{i}^{\prime}, \\
f_{s}^{\prime}=\frac{1}{h \pi} \int f(d \sigma)_{x}=W_{s} .
\end{gathered}
$$

Wie hier aus $f_{s}$ die Funktion $f_{s}^{\prime}$, so wird aus $f_{s}^{\prime}$ die Funktion $f_{s}^{\prime \prime}$ u. s. w. gebildet. Entsprechend bilde man die Potentiale

$$
\begin{array}{cc}
W_{i}=\frac{1}{h \pi} \int f\left(d \sigma_{i}\right), & W_{i s}=f_{s}^{\prime}+f_{s}, \\
W_{i}^{\prime}=\frac{1}{h \pi} \int f^{\prime}\left(d \sigma_{i}\right), & W_{i s}^{\prime}=f_{i}^{\prime \prime}+f_{s}^{\prime} . \\
\vdots & \vdots
\end{array}
$$

Herr C. Neumann beweist, dafs diese Funktionen $f_{s}, f_{s}^{\prime}, f_{s}^{\prime \prime}, \ldots$ gegen eine Konstante $C$ konvergieren:

$$
\lim _{n=\infty} f_{i}^{(n)}=C \text {. }
$$

Die Lösung für ein inneres Gebiet ist dann in der einen Form folgende:

$$
\Psi_{i}=C+\left(W_{i}-W_{i}^{\prime}\right)+\left(W_{i}^{\prime \prime}-W_{i}^{\prime \prime \prime}\right)+\cdots
$$

Bei der Anwendung auf den Kreis verfahre ich im ganzen nach der Vorschrift des Herrn C. Neumann, lasse aber eine Abänderung eintreten, welche jede andere Voraussetzung über die Randfunktion $f$, als dafs sie stetig ist, vermeidet. Man bilde nach der Vorschrift:

$$
W_{i}=\frac{1}{\pi} \int \dot{f}(d \sigma)_{i},
$$

$$
W_{i}=\frac{1}{\pi} \int_{i}^{2 \pi} f\left[1+\sum_{i}^{\infty}\left(\frac{\rho_{i}}{\rho}\right)^{n} \cos n\left(\omega-\omega_{i}\right)\right] d \omega .
$$

3

Universitäts- und

Landesbibliothek Düsseldorf 
Diese unter dem Integralzeichen stehende Reihe ist für jedes $\varrho_{i}<\varrho$ längs der ganzen Peripherie gleichmäfsig konvergent, woran auch durch die Multiplikation mit der endlichen und stetigen Funktion $f$ nichts geändert wird, so dafs die vorgeschriebene Integration gliedweise vorgenommen werden darf. Man erhält auf diese Weise:

$$
W_{i}=\frac{1}{\pi} \int_{0}^{2 \pi} f d \omega+\frac{1}{\pi} \sum_{1}^{\infty}\left(\frac{\rho_{i}}{\varrho}\right)^{n}\left\{\cos n \omega_{i} \int_{0}^{2 \pi} f \cos n \omega d \omega+\sin n \omega_{i} \int_{0}^{2 \pi} f \sin n \omega d \omega\right\} .
$$

Nun ist nach der Definition

$$
f_{x}^{\prime}=\frac{1}{\pi} \int f(d \sigma)_{s}=\frac{1}{\pi} \int f \frac{d \sigma}{2 \varphi}=\frac{1}{2 \pi} \int_{0}^{2 \pi} f d \omega=C .
$$

Die folgenden Funktionen $f^{\prime \prime}, f_{\text {s"', }}^{\prime \prime}$, werden im Falle des Kreises ebenfalls $=C$. Nach der Bedeutung von $W_{i}$, erhält man, wenn die Annäherung von $i$ an $s$ längs eines Radius geschieht,

$$
W_{i \pi}=\lim _{\rho=\rho}\left[\frac{1}{\pi} \int_{0}^{2 \pi} f d \omega+\frac{1}{\pi} \sum_{1}^{\infty} n\left(\frac{\rho_{i}}{\rho}\right)^{n}\left\{\cos n \omega_{i} \int_{0}^{2 \pi} f \cos n \omega d \omega+\sin n \omega_{i} \int_{0}^{2 \pi} f \sin n \omega d \omega\right\}\right] .
$$

Setzt man dies gemäls obiger Gleichung $=f_{s}^{\prime}+f_{s}$, so entsteht, wenn man noch die Gleiehung

berücksichtigt,

$$
f_{s}^{\prime}=\frac{1}{2 \pi} \int_{0}^{2 \pi} f \omega=C
$$

(A) $f_{s}=f(\omega)=\lim _{\beta_{i}=\rho}\left[\frac{1}{2 \pi} \int_{0}^{2 \pi} f d \omega+\frac{1}{\pi} \sum_{1}^{\infty}\left(\frac{\rho_{i}}{\varrho}\right)^{n}\left\{\cos n \omega \int_{0}^{2 \pi} f(\alpha) \cos n \alpha d \omega+\sin n \omega \int_{0}^{2 \pi} f(\alpha) \sin n \alpha d \alpha\right\}\right]$.

Es ist hervorzuheben, dafs der Limes $\rho_{i}=\rho$ erst nach Bildung der eckigen Klammer für $\varrho_{i}<\rho_{\text {zu }}$ nehmen ist. Wam es erlaubt ist, in der Klammer gliedweise $\varrho_{i}=\rho \mathrm{zu}$ setzen, wodurch die Fourier'sehe Reihe entsteht, darüber nachher.

Da der in $W_{i}$, geforderte Übergang eines inneren Punktes $\left(\varrho_{i}, \omega_{i}\right)$ in einen Peripheriepunkt $(\varrho, \omega)$ hier längs eines Radius geschah, so ist $\omega_{i}=\omega$ gesetzt worden. Die $\omega$ unter dem Zeichen $j$ sind zum Unterschiede mit $\alpha$ vertauscht. Das innere Problem für den Kreis wird, da $W_{i}^{\prime}=W_{i}^{\prime \prime}=\cdots \cdot=2 C$, gelöst durch

$$
\psi_{i}=-C+W_{i}=-\frac{1}{2 \pi} \int_{0}^{2 \pi} f d \omega+\frac{1}{\pi} \int_{0}^{2 \pi} f(d \sigma)_{i} .
$$

Diese beiden Gleichungen für $f_{\sigma}=f(\omega)$ und für $\psi_{\text {; }}$ gelten, ohne dafs ein Ansatz für $f$ geschehen ist, also für jede stetige Funktion ohne Einschränkung. Speziell giebt die Gleichung (A) eine Darstellung, welche, eben nach der Methode des arithmetischen Mittels, für jede stetige Funktion gilt, auch wenn die entsprechende Fourier'sche Reihe nicht konvergiert. Diese Art der Darstellung ist nur eine nicht übliche; sie erfordert nämlich als letzte Grenzoperation die Bildung eines Limes für $r=1-0$, (wenn von jetzt an $\frac{Q_{i}}{Q}=r$ gesetzt wird), während sonst die Form der unendlichen Reihe oder des Integrals bevorzugt ist. Man ersieht aus (A), wern es etwas anders geschrieben wird: Es ist für jede stetige Funktion unter allen Umständen:

$\lim _{r=1-0}\left\{\lim _{n=\infty}\left[\frac{1}{2 \pi} \int_{0}^{2 \pi} f(\alpha) d \alpha+\frac{1}{\pi} \sum_{1}^{n} r^{n}\left(\cos n \omega \int_{0}^{2 \pi} f(\alpha) \cos n \alpha d \alpha+\sin n \omega \int_{0}^{2 \pi} f(\alpha) \sin n \omega d \alpha\right)\right]\right\}=f(\omega)$. 
$-19-$

Hingegen giebt es Fälle, wo

$$
\lim _{n=\infty}\left\{\lim _{r=1}[\text { idem] }\} \text { d. i. } \frac{1}{2 \pi} \int_{0}^{2 \pi} f(\alpha) d \alpha+\frac{1}{\pi} \sum_{1}^{\infty} \int_{0}^{2 \pi} f(\alpha) \cos n(\omega-\alpha) d \alpha\right.
$$

die stetige Funktion nicht darstellt. Ersteres erkennt man hier aus der C. Neumann'schen Methode, letzteres ist bekannt aus der Theorie der Fourier'schen Reihe. - Da die Fourier'sche Reihe hier als Potenzreihe, gebildet für $r=1$, auftritt, so ist an den Abel'schen Satz und die Verallgemeinerung desselben zu erinnern, derselbe lautet*): Sei

$$
\varphi(r)=a_{0}+a_{1} r+a_{2} r^{2}+\cdots
$$

eine Potenzreihe mit der Konvergenzgrenze $r=1$. Wenn dann die Reihe $a_{0}+a_{1}+a_{2}+\cdots$ nach einer endlichen Zahl konvergiert oder bestimmt unendlich ist, so ist $\lim _{r=1-0} \varphi(r)=$ dieser endlichen bezw. bestimmt unendlichen Zahl. Hat dagegen $a_{0}+a_{1}+a_{2}+\cdots$ die Unbestimmtheitsgrenzen $O$ und $U$, ferner $\lim _{r=1-0} \varphi(r)$ die Unbestimmtheitsgrenzen $O^{\prime}$ und $U^{\prime}$, so liegen dieselben, ob endlich oder unendlich, so zu einander:

$$
U \leqq U^{\prime} \leqq O^{\prime} \leqq 0 .
$$

Aus diesem Satze folgt nach dem obigen: Wenn die Fourier'sche Reihe (gebildet von einer durchweg stetigen Funktion) konvergiert, so konvergiert sie nach dem. Funktionswert. Konvergiert sie nicht, so schwankt sie um den Funktionswert herum. Hier ist nämlich $U^{\prime}=O^{\prime}=f(\omega)$, also $U \leqq f(\omega) \leqq 0$. Das Entsprechende gilt von dem Problem für die Kugel und der Entwicklung nach Kugelfunktionen.

Wenn man auf die durchgängige Stetigkeit der Funktion $f$ verzichtet, so wird die oben (S. 16) gegebene Definition der Fundamentalfunktion, wenigstens was den einen Punkt, die Stetigkeit in Erstreckung des ganzen Gebietes, anbetrifft, hinfällig, während die gegebene Lösung, so lange die Funktion integrierbar bleibt, ihren Sinn behält. Es entsteht nun die Aufgabe, zu untersuchen, welches modifizierte Randwertproblem die frühere Lösung bei der jetzigen Voraussetzung löst, d. h. zu untersuehen, wie jetzt der Übergang in die Randwertfunktion, wiederum zunächst längs eines Radius, stattfindet. Die Funktion $f$ werde also von jetzt an lediglich als integrierbar vorausgesetzt, sie möge auch in unendlich vielen Punkten eines Systems erster Gattung unendlich werden können, doch so, da/s auch ihr absoluter Wert integrierbar ist. Zu untersuchen ist der Limes auf der rechten Seite der Gleichung (A).

Die Reihe in der eckigen Klammer konvergiert offenbar für jedes $\rho_{i}<\varrho_{\text {bezw. } r<1, \text { da }}$ nach der gemachten Voranssetzung, wie ich als bekannt annehme,

$$
\left.\lim _{n=\infty} \int_{0}^{2 \pi} f(\alpha) \cos n \alpha d \alpha \quad \text { und } \quad \lim _{n=\infty} \int_{0}^{2 \pi} f(\alpha) \sin n \alpha d \alpha=0 \text {. } * \alpha\right)
$$

Da ferner, wenn $r<1$, die Reihe

$$
\frac{1}{2}+\sum_{1}^{\infty} r^{n} \cos n(\omega-\omega)
$$

für alle « gleichmäfsig konvergiert, so darf man die Ordnung von Summation und Integration vertauschen (vgl. Dini, Grundlagen $\$ 286$ ) und erhält, falls die eben erwähnte Reihe als reeller Teil der folgenden

$$
\frac{1}{2}+\sum_{1}^{\infty} r^{n} e^{i n(\omega-\alpha)}
$$

betrachtet und summiert wird, folgende Gleichung:

*) Stolz, Arithm, I, S. 280.

*8) Harnack, Math. Ann. 19, S. 268. Es ist übrigens durchaus nicht erforderlich, dafs diese Integrale gerade Null werden.

$3^{*}$

Universitaits- und

Landesbibliothek Düsseldorf 
(C) $\frac{1}{2 \pi} \int_{0}^{2 \pi} f(\alpha) d \alpha+\frac{1}{\pi} \sum_{1}^{\infty} r^{n}\left(\cos n \omega \int_{0}^{9 \pi} f(\alpha) \cos n \alpha d \alpha+\sin n \omega \int_{0}^{2 \pi} f(\alpha) \sin n \alpha d \alpha\right)=\frac{1}{2 \pi} \int_{0}^{2 \pi} f(\alpha) \frac{1-r^{2}}{1-2 r \cos (\omega-\alpha)+r^{2}} d \alpha$, welche gilt für $r<1 . *$ ) -

Unter den über $f$ gemachten Voraussetzungen haben beide Seiten der Gleichung (C) bestimmte Werte und sind einander gleich, wie unter Berufung auf das Verschwinden obiger Integrale für $n=\infty$ und den Satz bei Dini, welcher die Umkehrung der Summations- und Integrationsordnung gestattet, bewiesen worden ist. Bei den allerallgemeinsten Annahmen über das Unendlichwerden der Funktion $f$, wie sie speziell Harnack einführt und begrifflich hiervon verschieden aber auf dasselbe herauskommend Herr Holder**), ist die obige Schlufsweise nicht bindend und die Gleichnng (C) kann nicht als bewiesen angesehen werden. Kann man doch bei dieser allgemeinsten Art des Unendlichwerdens einer integrierbaren Funktion nicht ohne weiteres auf die Integrierbarkeit des Produkts dieser Funktion mit einer endlichen und integrierbaren, z. B. auf die Integrierbarkeit von $f(\alpha) \frac{\cos n \alpha}{\sin n \alpha}$ schliefsen. Sofern bei andern Voraussetzungen als den obigen die Gleichung (C) ebenfalls gilt, gelten auch die folgenden Schlüsse. -

Das Poisson'sche Integral, welches nach (C) die Potenzreihe für jedes $r<1$ summiert, wird nun dazu benutzt, um den Limes dieser Potenzreihe für $r=1-0$ zu bestimmen, am einfachsten in der Weise du Bois-Reymonds $\left.{ }^{* * * *}\right)$.

Wir bedienen uns der Eigenschaft von

$$
\frac{1-r^{2}}{1-2 r \cos (\omega-\alpha)+r^{2}}
$$

stets positiv zu sein für $r<1$ und der Gleichung

$$
\int_{0}^{2 \pi} d \epsilon \frac{1-r^{2}}{1-2 r \cos (\omega-\alpha)+r^{2}}=2 \pi .
$$

Jedenfalls ist der Limes $(r=1)$ dieses Integrals ebenfalls $2 \pi$. Zerlegt man es in:

$$
\int_{0}^{0-\delta}+\int_{i=\delta}^{0+\delta}+\int_{\omega+\delta}^{2 \pi}=2 \pi
$$

so ist der Limes $(r=1)$ der beiden äufseren Integrale links Null (wegen des Faktors 1 - $r^{2}$ und der Endlichkeit der Funktion unter dem Integralzeichen für $r=1$ incl.), also ist der des mittleren allein $2 \pi$. Zerlegt man dieses in die Teile

$$
\int_{\omega-\delta}^{\infty}+\int_{\infty}^{\omega+\delta}
$$

so hat jeder für sich, weil sie ganz gleich sind, den Limes $\pi$ für $r=1$. Wir bilden nunmehr

$$
\int_{0}^{2 \pi} f(\alpha) \frac{1-r^{2}}{1-2 r \cos (\omega-\alpha)+r^{2}} d \alpha=\int_{0}^{\omega-\delta}+\int_{\omega+\delta}^{2 \pi}+\int_{\omega-\delta}^{\omega}+\int_{\omega}^{\varphi+j} .
$$

Auf die beiden letzten Integrale wird der erste Mittelwertsatz angewandt. Auch in dem vorliegenden Falle, dafs die Funktion $f$ unendlich wird, ist

*) Diese Methode findet sich zuerst bei Herrn C. Neumann (Crelle 59), dann bei den Herren Prym und Schwarz (daselbst 73 und 74); ferner bei Harnack (Math. Ann, 21, S. 322).

*) beides in Math. Ann, 24

wer) Crelle 103 , S. 216. 
$-21-$

$$
\int_{a}^{b} f(\alpha) \frac{1-r^{2}}{1-2 r \cos (\omega-\alpha)+r^{2}} d \alpha=[f(\alpha)]_{a}^{b} \int_{a}^{b} \frac{1-r^{2}}{1-2 r \cos (\omega-\alpha)+r^{2}} d \alpha .
$$

Hierin bedeutet der erste Faktor rechts einen unbekannten Mittelwert, gleichgiltig, ob die Funktion diesen Wert wirklich annimmt oder nicht. Dieser Mittelwert mufs endlich sein, weil beide Integrale rechts und links bestimmt sind, das rechte positiv. Hierdurch entsteht:

$$
\int_{0}^{2 \pi} f(\alpha) \frac{1-r^{2}}{1-2 r \cos (\omega-\alpha)+r^{2}} d \omega=\int_{0}^{\omega-\delta}+\int_{\omega+\delta}^{2 \pi}+[f(\alpha)]_{\omega-\delta}^{\omega} \int_{\omega-\delta}^{\omega} \frac{1-r^{2}}{1-2 r \cos (\omega-\alpha)+r^{2}} \cdot d \alpha+[f(\alpha)]_{\omega}^{\omega+\delta} \int_{\omega}^{\omega+\delta} .
$$

Hiervon ist der Limes für $r=1$ zu bilden. $\delta$ bleibt hierbei fest und zu späterer Verfügung. Die beiden letzten Integrale haben, wie gezeigt, den Grenzwert $\pi$; es ist zu zeigen, dafs die beiden ersten gegen 0 konvergieren. Den Faktor $1-r^{2}$ ziehe man vor das Integral. Für jedes $\alpha$ im Intervall des ersten sowie des zweiten Integrals ist für ein festes $r$

ferner ist für alle $r>\cos \delta$

$$
\frac{1}{1-2 r \cos (\omega-\alpha)+r^{2}}<\frac{1}{1-2 r \cos \delta+r^{2}},
$$

$$
\frac{1}{1-2 r \cos \delta+r^{2}}<\frac{1}{1-\cos ^{2} \delta}=\frac{1}{\sin ^{2} \delta} \text {. }
$$

Daraus folgt $(f(c)$ ist $\mathrm{n}$. V. absolut integrierbar)

$$
\left|\int_{0}^{\omega-\delta} f(\alpha) \frac{1}{1-2 r \cos (\omega-\alpha)+r^{2}} d \alpha\right|<\frac{1}{\sin ^{2} \delta} \int_{0}^{\omega-\delta}|f(\alpha)| d \alpha .
$$

Da die Grölse rechts endlich ist, so ist ersichtlich, wie der Limes des ersten, und ebenso des zweiten Integrals rechts, wegen des Faktors $1-r^{2}$ verschwindet. Man erhält also

$$
\lim _{r=1-0} \int_{0}^{2 \pi} f(\alpha) \frac{1-r^{2}}{1-2 r \cos (\omega-\alpha)+r^{2}} d \alpha=\pi\left\{[f(\kappa)]_{\omega-\delta}^{\omega}+[f(\kappa)]_{\omega}^{\omega+\delta}\right\} .
$$

Rechts darf man nunmehr das willkürliche $\delta$ gegen Null konvergieren lassen und erhält den Grenswert des Poisson'schen Integrals besw. der gleichwertigen Potensreihe (s. Gleichung (C))

$$
=\frac{1}{2}\{f(\omega-0)+f(\omega+0)\} \text {. }
$$

Hierin bedeutet $f(\omega-0)$ einen bestimmten oder unbestimmten Wert innerhalb des Wertvorrats zur linken Seite, $f(\omega+0)$ das Entsprechende zur rechten Seite. Auf den Funktionswert in $\omega$ selbst kommt es beide Male nicht an. -

Drei Ausdricke sind es also, deren Verhalten zu einander beobachtet werden soll:

1) Die Funktion $f(\omega)$.

2) Der Limes des Poisson'schen Integrals besw. der gleichwertigen Potenzreihe (Gleichung (C)) für $r=1-0$.

3) Die Fourier'sche Reihe, d. i. die Potensreihe gebildet für den Wert $r=1$.

Was zunächst die Funktion unter 1) anbetrifft, so denke man sich dieselbe von hebbaren Unstetigkeiten, welche ganz einflufslos sind, befreit und unter Umständen auch durch Hinzufügung einer Funktion vom Integral Null vereinfacht. Die so modifizierte Funktion nenne man $f$; sie habe in $\omega$ die vorderen Unbestimmtheitsgrenzen $V_{o}$ und $V_{u}$, die hinteren $H_{0}$ und $H_{u}$. - Die Grenzen des Limes in 2) seien wieder $O^{\prime}$ und $U^{\prime}$, die der Fourier'schen Reihe in 3) $O$ und $U$. Wir erhalten dann folgendes Schema:

$$
\begin{gathered}
U \leqq U^{\prime} \leqq O^{\prime} \leqq 0 \\
\frac{V_{u}+H_{u}}{2} \leqq U^{\prime} \leqq O^{\prime} \leqq \frac{V_{o}+H_{o}}{2},
\end{gathered}
$$

Universitäts- und

Landesbibliothek Düsseldorf 
in welchem (a) aus dem Potenzreihensatz (S. 19), (b) aus Gleichung (D) folgt. In (b) verstärkt man unter Umständen die Ungleichung, wenn man links die untere, rechts die obere Schranke des Wertvorrats der Funktion $f$ in $\omega$ einsetzt. Man sieht, dafs dieser Wertvorrat und das Unbestimmtheitsintervall der Fourier'schen Reihe sicherlich, wenn $U^{\prime}=O^{\prime}$, diesen Punkt, andernfalls das Stäck zwischen $U^{\prime}$ und $O^{\prime}$ gemeinsam haben. -

Hat $f$ in $\omega$ eimen vorderen Grenzwert $V$ und einen hinteren Grenzwert $H$, so folgt aus unserm Schema, da $V_{o}=V_{u}=V$ und $H_{0}=H_{u}=H$ ist,

$$
\text { - } U^{\prime}=O^{\prime}=\frac{V+H}{2}
$$

weiter aus (a), dafs die Fourier'sche Reihe $(O, U)$ genau diesen Wert darstellt oder um ihn herumschwankt.

Ist $f(\omega)$ bestimmt unendlich, etwa $+\infty$, so ist $V_{u}=H_{u}=V_{o}=H_{o}=+\infty$, also auch $O^{\prime}=U^{\prime}=+\infty$, demzufolge auch $O=+\infty$, während über $U$ nichts folgt. Ist also die Funktion $f$ in $\omega+\infty(-\infty)$, so ist die obere (untere) Unbestimmtheitsgrenze der entsprechenden Fourier'sehen Reihe gleichfalls $+\infty(-\infty)$. Ist $f$ bei $\omega$ so beschaffen, dafs die Werte in einer beliebig kleinen Umgebung rechts und links symmetrisch und entgegengesetzt bezeichnet sind, so folgt aus der Herleitung (s. S. 21), weil die Mittelwerte vor den beiden letzten Integralen rechter Hand entgegengesetzt und diese Integrale selbst gleich sind, dafs Null herauskommt. Dies ist auch der Fall, wenn $\omega$ ein Unendlichkeitspunkt ist. Es ist dann $U^{\prime}=O^{\prime}=0$ und die Fourier'sche Reihe ist $=0$ oder schwankt um 0 herum. -

Macht man umgekehrt eine Annahme in Betreff der Fourier'schen Reihe, so kann man einen Schluf's auf die Funktion ziehen. Ist die Fourier'sche Reihe konvergent, also $O=U$, so folgt $O^{\prime}=U^{\prime}$ und weiter, dal's die zu Grunde liegende Funktion denselben Wert hat, oder um diesen Wert (möglicherweise zwischen den Grenzen $+\infty$ und $-\infty$ ) herumschwankt. - 
in welchem (a) ans dem Potenzr unter Umständen die Ungleichun vorrats der Funktion $f$ in $\omega$ ein intervall der Fourier'sehen Reih. zwischen $U^{\prime}$ und $O^{\prime}$ gemeinsam Hat $f$ in $\omega$ eimen vord unserm Schema, da $V_{0}=V_{u}=$

weiter aus (a), dals die Fourier' schwankt.

Ist $f(\omega)$ bestimmt unen $O^{\prime}=U^{\prime}=+\infty$, demzufolge au in $\omega+\infty(-\infty)$, so ist die ob Reihe gleichfalls $+\infty(-\infty)$. Umgebung rechts und links sym leitung (s. S. 21), weil die Mittel und diese Integrale selbst gleich Unendlichkeitspunkt ist. Es ist c um 0 herum. -

Macht man umgekehrt Schluf's auf die Funktion ziehen. und weiter, dafs die zu Grunde licherweise zwischen den Grenze
$-29-$

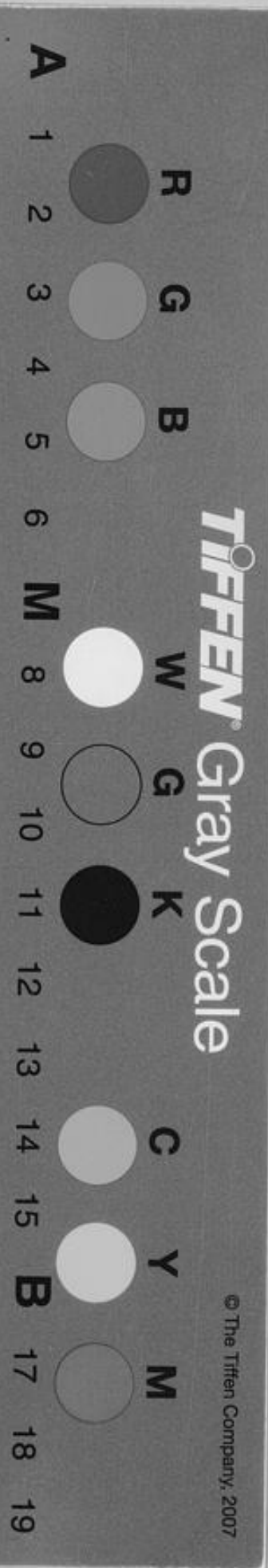

b) folgt. In (b) verstärkt man die obere Schranke des Wertrat und das UnbestimmtheitsPunkt, andernfalls das Stück

in Grenzwert $H$, so folgt aus

darstellt oder um ihn herum-

$V_{0}=H_{0}=+\infty$, also auch folgt. Ist also die Funktion $f$ $r$ entsprechenden Fourier'schen Werte in einer beliebig kleinen ret sind, so folgt aus der Hern rechter Hand entgegengesetzt ist auch der Fall, wenn $\omega$ ein e Reihe ist $=0$ oder schwankt

hen Reihe, so kann man einen also $O=U$, so folgt $O^{\prime}=U^{\prime}$ t, oder um diesen Wert (mög- 